\title{
Sharp asymptotics for the quasi-stationary distribution of birth-and-death processes
}

\author{
J.-R. Chazottes ${ }^{(a)}$, P. Collet ${ }^{(a)}$, S. Méléard ${ }^{(b)}$ \\ (a) Centre de Physique Théorique, CNRS UMR 7644, Ecole polytechnique \\ F-91128 Palaiseau Cedex (France) \\ (b) Centre de Mathématiques Appliquées, CNRS UMR 7641, Ecole polytechnique \\ F-91128 Palaiseau Cedex (France) \\ June 18, 2018
}

\begin{abstract}
We study a general class of birth-and-death processes with state space $\mathbb{N}$ that describes the size of a population going to extinction with probability one. This class contains the logistic case. The scale of the population is measured in terms of a 'carrying capacity' $K$. When $K$ is large, the process is expected to stay close to its deterministic equilibrium during a long time but ultimately goes extinct. Our aim is to quantify the behavior of the process and the mean time to extinction in the quasi-stationary distribution as a function of $K$, for large $K$. We also give a quantitative description of this quasi-stationary distribution. It turns out to be close to a Gaussian distribution centered about the deterministic long-time equilibrium, when $K$ is large.

Our analysis relies on precise estimates of the maximal eigenvalue, of the corresponding eigenvector and of the spectral gap of a self-adjoint operator associated with the semigroup of the process.
\end{abstract}




\section{Contents}

1 Introduction 3

2 Standing assumptions and notations 5

3 Statements of the main results 7

3.1 The generator and its spectrum .............

3.2 Estimates of the largest eigenvalue and of the associated eigenvector . . . . . . . . . . . . . . . . . 9

3.3 Quasi-stationary distribution, survival rate and mean time to extinction .................... 10

3.4 Convergence rate to the quasi-stationary distribution and Gaussian approximation . . . . . . . . . . . . . .

4 Proof of Theorem $3.1 \quad 13$

$4.1 \quad \tilde{L}$ is symmetric and closable in $\ell^{2}(\pi) \ldots \ldots \ldots \ldots$

$4.2 L$ defines a positive contraction semigroup in $\ell^{2}(\pi) \ldots \ldots . \quad 14$

4.3 Compactness, self-adjointness and dissipativity . . . . . 17

5 Proof of Theorem $3.2 \quad 18$

5.1 When $1 \leq n \leq n_{*}(K) \ldots \ldots \ldots \ldots \ldots \ldots$

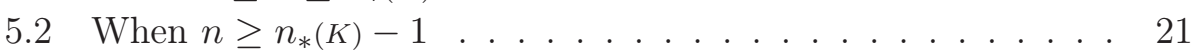

5.3 Matching. . . . . . . . . . . . . . . . . 22

6 Proof of Theorem $3.3 \quad 25$

6.1 A Poincaré inequality . . . . . . . . . . . . 25

6.2 Lower estimate for the spectral gap . . . . . . . . . . . . 29

7 Proof of Theorem $3.6 \quad 33$

7.1 Preliminary estimates $\ldots \ldots \ldots \ldots \ldots$

7.2 Proof of Theorem $3.6 \ldots \ldots \ldots$

8 Proof of Theorem $3.7 \quad 38$

9 Appendix: some technical lemmas and estimates 39 


\section{Introduction}

We study a general class of birth-and-death processes with state space $\mathbb{N}$ that describes the size of a population going to extinction with probability one. For a population of size $n \in \mathbb{N}^{*}$, the birth rate is denoted by $\lambda_{n}>0$ and the death rate by $\mu_{n}>0$. Furthermore, we assume that

$$
\lambda_{n}^{K}=n \tilde{\lambda}\left(\frac{n}{K}\right), \quad \mu_{n}^{K}=n \tilde{\mu}\left(\frac{n}{K}\right)
$$

where $\tilde{\lambda}, \tilde{\mu}$ are positive functions and $K$ is a scaling parameter describing the amount of available resources (that is called the 'carrying capacity' in ecology). We assume that $\lambda_{0}=\mu_{0}=0$, entailing absorption at state 0 .

In this work, we consider the case where absorption at 0 happens with probability one. We also assume that the time to this absorption has finite expectation. In this situation, the unique stationary probability measure is $\delta_{0}$, the Dirac mass at state 0 . In order to understand the behavior of the process before absorption, a relevant object to look at is a so-called quasistationary distribution, i.e, a probability distribution that is stationary when the process is conditioned to survive. Our aim is to describe what happens for large $K$.

The prominent example is the so-called logistic birth-and-death process $\left(X_{t}^{K}, t \geq 0\right)$ defined by following birth and death rates

$$
\lambda_{n}^{K}=\tilde{\lambda} n, \mu_{n}^{K}=n\left(\tilde{\mu}+\frac{n}{K}\right)
$$

for $n \geq 1$, where $\tilde{\lambda}, \tilde{\mu}$ are positive parameters. It is a classical result (see e.g. [16]) that if the process starts in a state of the form $\left\lfloor x_{0} K\right\rfloor\left(x_{0}>0\right)$, then the rescaled process $X_{t}^{K} / K$ is 'close', in the limit as $K \rightarrow \infty$, during any given finite interval of time, to the solution of the differential equation

$$
\frac{\mathrm{d} x}{\mathrm{~d} t}=x(\tilde{\lambda}-\tilde{\mu}-x)
$$

with initial condition $x_{0}$. This differential equation has a unique attractive equilibrium $x_{*}=\tilde{\lambda}-\tilde{\mu}$ and the integer $\left\lfloor x_{*} K\right\rfloor$ can be considered as an approximation of the population size over every given finite time interval. However, for each $K$, the process $X_{t}^{K}$ goes almost surely to extinction as $t \rightarrow \infty$, see [10].

In this paper, we consider more general processes with the same kind of behavior. One of our motivations is to quantify, as a function of $K$, the scale of the mean time to extinction, the time-scale of convergence to the quasi-stationary distribution, and the time-scale during which the process is close to the rescaled deterministic equilibirum $\left\lfloor x_{*} K\right\rfloor$ with high probability.

Our results can be colloquially described as follows. We get an upper bound of order $K \log K$ for the time it takes for the process to be close to 
the quasi-stationary distribution. We also get the existence of a time interval, exponentially long in $K$, during which the process, if we start from a population of order $K$, is nearly distributed according to the quasi-stationary distribution.

We also prove that the total variation distance between the quasi-stationary distribution and a Gaussian distribution is bounded by $1 / \sqrt{K}$. This Gaussian distribution is centered around $\left\lfloor x_{*} K\right\rfloor$ and its variance is of order $K$.

As a by-product of our analysis we show that the mean time to extinction with respect to the quasi-stationary distribution is given by

$$
\frac{c}{\sqrt{K}} e^{K \int_{0}^{x_{*}} \log \frac{\tilde{\lambda}(x)}{\tilde{\mu}(x)} \mathrm{d} x}\left(1+\mathcal{O}\left(\frac{(\log K)^{3}}{\sqrt{K}}\right)\right)
$$

where $c$ is a constant independent of $K$ that is explicitly given later on. Roughly speaking, this mean time is exponentially large in $K$.

Motivated by population extinction in biology, many people attempted to analyze quasi-stationary distributions. But even in the simplest models, like the logistic model, this turned out to be a complicated task. Previous results are mostly based on either Monte-Carlo simulations or uncontrolled approximations based on heuristic ansatzes, see the review paper [20] and also [19, 15]. The present work is the first one in which controlled mathematical approximations are obtained for the quasi-stationary distribution for a class of models encompassing the logistic model.

We are aware of only a few mathematical results related to our work. In [9], the authors do not study the quasi-stationary distribution but only the mean time to expectation starting from a state of order $K$ for which they obtain the asymptotic behavior in $K$ (see also [21]). Here we are able to control this quantity for all initial states and also for the quasi-stationary distribution as a starting distribution. In [2], the authors show that the quasistationary distribution can be approximated in total variation distance by an auxiliary process called the 'returned process'. They also prove a bound for the total variation distance between the law of the process $X_{t}^{K}$ for fixed values of $t$ and the quasi-stationary distribution. This is somewhat related to one of our theorems (Theorem 3.6). Let us also mention the articles [5, 6, 8] about quantitative convergence to quasi-stationarity.

The main tool in this work is the analysis of an operator $L$ that is related to the generator of the killed process. We use a weighted Hilbert space where $L$ is self-adjoint. The operator $L$ has a maximal simple and negative eigenvalue $-\rho_{0}$. The mean time to extinction is exactly $1 / \rho_{0}$. The quasi-stationary distribution is constructed from the corresponding positive eigenvector. The method of analysis of the equation $L u=-\rho_{0} u$ is inspired by matching techniques reminiscent of the WKB method in Physics [11, 17]. 


\section{Standing assumptions and notations}

In the sequel most quantities will depend on the parameter $K$. We will not indicate systematically this dependence in the notation, except when we want to highlight it. Recall that

$$
\lambda_{n}=n \tilde{\lambda}\left(\frac{n}{K}\right), \mu_{n}=n \tilde{\mu}\left(\frac{n}{K}\right) .
$$

In the rest of the paper, the functions $x \mapsto \tilde{\lambda}(x)$ and $x \mapsto \tilde{\mu}(x)$, defined on $\mathbb{R}_{+}$, are assumed to be positive, differentiable and increasing. In particular, this implies that the sequences $\left(\lambda_{n}\right)_{n}$ and $\left(\mu_{n}\right)_{n}$ are increasing.

From now on, we assume that the following properties for the functions $\tilde{\lambda}$ and $\tilde{\mu}$ hold throughout the paper.

- $\lim _{x \rightarrow+\infty} \frac{\tilde{\lambda}(x)}{\tilde{\mu}(x)}=0$;

- $\tilde{\lambda}(0)>\tilde{\mu}(0)>0$;

- There exists a unique $x_{*} \in \mathbb{R}_{+}$such that $\tilde{\lambda}\left(x_{*}\right)=\tilde{\mu}\left(x_{*}\right)$;

- $\tilde{\lambda}^{\prime}\left(x_{*}\right) \neq \tilde{\mu}^{\prime}\left(x_{*}\right)$;

- $\int_{\frac{x_{*}}{2}}^{+\infty} \frac{\mathrm{d} x}{x \tilde{\mu}(x)}<+\infty$;

- $\sup _{x \in \mathbb{R}_{+}} \frac{\tilde{\mu}^{\prime}(x)}{\tilde{\mu}(x)}<+\infty$;

- The function $x \mapsto \log \frac{\tilde{\mu}(x)}{\tilde{\lambda}(x)}$ defined on $\mathbb{R}_{+}$is increasing.

The function $H: \mathbb{R}_{+} \rightarrow \mathbb{R}$ defined by

$$
H(x)=\int_{x_{*}}^{x} \log \frac{\tilde{\mu}(s)}{\tilde{\lambda}(s)} \mathrm{d} s
$$

is assumed to have the following properties:

- $H$ is three times differentiable;

- $\sup _{x \in \mathbb{R}_{+}}\left(1+x^{2}\right)\left|H^{\prime \prime \prime}(x)\right|<+\infty$.

Some comments are in order about the above assumptions. The relevant assumptions from a biological viewpoint are assumptions (2.2), (2.3) and (2.4). The first one means that, when the population size gets large, deaths prevail. The second one means the opposite: at low population size, births prevail. The third one means that there is a unique equilibrium for the associated differential equation. This rules out for instance the so-called Allee effect where there are two non-trivial equilibria. Assumption (2.5) is a genericity property. The remaining assumptions are technical but they are by far true in the logistic case and in many other models. 
We shall denote by $\left(X_{t}^{K}, t \geq 0\right)$ the birth-and-death process associated with the rates $\left(\lambda_{n}\right)$ and $\left(\mu_{n}\right)$. Thorough the paper we will use the classical notation

$$
\pi_{n}=\frac{\lambda_{1} \cdots \lambda_{n-1}}{\mu_{1} \cdots \mu_{n}}, \text { for } n \geq 2
$$

and we set $\pi_{1}:=\frac{1}{\mu_{1}}$. The following trivial identity will be used repeatedly.

$$
\lambda_{n} \pi_{n}=\mu_{n+1} \pi_{n+1} .
$$

One can verify that condition (2.2), together with the facts that $\left(\mu_{n}\right)_{n}$ is increasing and that $\tilde{\mu}(0)$ is bounded away from zero, imply the following two properties:

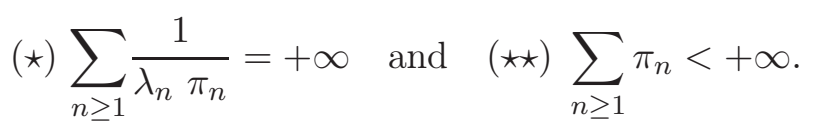

The property $(\star)$ implies absorption of the process at state 0 with probability one. The property $(\star \star)$ ensures finiteness of the expectation of the absorption time, that is, $\mathbb{E}_{m}\left[T_{0}\right]<+\infty$ for every $m \in \mathbb{N}^{*}$, where $T_{0}=\inf \left\{t \geq 0: X_{t}^{K}=\right.$ $0\}$. We refer to [13, p. 384] and [1, chapter 3] for details.

Condition (2.6) implies

$$
\sum_{n \geq 1} \frac{1}{\lambda_{n} \pi_{n}}\left(\sum_{i \geq n+1} \pi_{i}\right)<+\infty .
$$

(See Lemma 9.1 for a proof.) As proved in [10, this is a sufficient condition for the existence and uniqueness of a quasi-stationary distribution. It turns out that it is a necessary condition as well as it can be deduced from [4]. Condition (2.7) implies

$$
\sup _{n} \frac{\mu_{n+1}}{\mu_{n}}<\infty .
$$

This follows from the mean value theorem to the function $x \mapsto \log \tilde{\mu}(x)$. We will assume that

$$
\pi_{n} \mu_{n}^{2} \stackrel{n \rightarrow \infty}{\longrightarrow} 0 .
$$

This is a technical condition that we use in the spectral theory of the operator associated with the process.

Finally, let us recall (see e.g. [16]) that for large $K$, the process $\left(X_{t}^{K} / K, t \geq\right.$ 0 ) is close to the solution of the ordinary differential equation

$$
\frac{\mathrm{d} x}{\mathrm{~d} t}=x(\tilde{\lambda}(x)-\tilde{\mu}(x))
$$

during any given finite time interval. Our assumptions imply that the differential equation (2.16) has the unique non-zero equilibrium $x_{*}$. Observe that, because of assumptions on the functions $x \mapsto \tilde{\lambda}(x)$ and $x \mapsto \tilde{\mu}(x)$, one 
has $\frac{\tilde{\lambda}(x)}{\tilde{\mu}(x)}>1$ for $x<x_{*}$ and $\frac{\tilde{\lambda}(x)}{\tilde{\mu}(x)}<1$ for $x>x_{*}$. This implies the stability of the equilibrium $x_{*}$ of the deterministic equation (2.16) and, using (2.5), we get

$$
H^{\prime \prime}\left(x_{*}\right)>0
$$

We shall use the notation

$$
n_{*}(K)=\left\lfloor x_{*} K\right\rfloor .
$$

This quantity plays a natural role in the sequel.

An example. For the logistic birth-and-death process defined in (1.1), we have $\tilde{\lambda}(x)=\tilde{\lambda}$ and $\tilde{\mu}(x)=\tilde{\mu}+x$. If $\tilde{\lambda}>\tilde{\mu}$, it is easy to check that all the above conditions are fullfilled. One has $n_{*}(K)=\lfloor(\tilde{\lambda}-\tilde{\mu}) K\rfloor$.

\section{Statements of the main results}

\subsection{The generator and its spectrum}

Our goal is to link the semigroup of the process $\left(X_{t}^{K}, t \geq 0\right)$ 'killed' at 0 to a self-adjoint operator with compact resolvent in an appropriate Hilbert space. The spectral theory for this operator lies at the core of our work.

Let us denote by $\mathscr{D}$ the set of sequences with finite support on $\mathbb{N}^{*}$. Define the operator $\tilde{L}$ with domain $\mathscr{D}$ by

$$
(\tilde{L} u)_{n}=\lambda_{n} u_{n+1}+\mu_{n} u_{n-1} \mathbb{1}_{\{n \geq 2\}}-\left(\lambda_{n}+\mu_{n}\right) u_{n} .
$$

We introduce the following weighted space of sequences of complex numbers

$$
\ell^{2}(\pi)=\left\{u=\left(u_{n}\right)_{n \in \mathbb{N}^{*}}: \sum_{n=1}^{\infty} \pi_{n}\left|u_{n}\right|^{2}<\infty\right\}
$$

where the $\pi_{n}$ 's are defined in (2.10). The space $\ell^{2}(\pi)$ is a Hilbert space when endowed with the scalar product

$$
\langle u, v\rangle_{\pi}=\sum_{n=1}^{\infty} \pi_{n} \bar{u}_{n} v_{n}
$$

where $\bar{u}_{n}$ is the complex conjugate of $u_{n}$. We shall denote by $\|\cdot\|_{\pi}$ the associated norm.

The main content of the following theorem is that one can extend the operator $\tilde{L}$ to an operator $L$ that is the infinitesimal generator of a positive and contractive semigroup in $\ell^{2}(\pi)$. Moreover, this operator has a discrete spectrum with a maximal eigenvalue that is simple and negative.

Theorem 3.1 (The operator $L, \rho_{0}, \varphi$ and $\rho_{1}$ ).

1. The operator $\tilde{L}$ is symmetric on $\mathscr{D}$. It is closable in $\ell^{2}(\pi)$. 
2. We will denote by $L$ its closure and by $\mathcal{D}$ the domain of this closure. The operator $L$ defines a positive contraction semigroup in $\ell^{2}(\pi)$.

3. $L$ is a dissipative, self-adjoint operator with a compact resolvent. Its spectrum is discrete and the maximal eigenvalue is simple and negative. We denote it by $-\rho_{0}$. The corresponding eigenvector can be chosen positive and we denote it by $\varphi$. Finally, we denote by $-\rho_{1}$ the second largest eigenvalue.

The proof of this theorem is given in Section 4.

REMARK 3.1. The construction of $\mathcal{D}$ is general; see [14, III.5.3].

For all $t>0, n, m \in \mathbb{N}^{*}$, let

$$
P_{t}(n, m)=\frac{1}{\pi_{n}}\left\langle\mathrm{e}_{n}, e^{t L} \mathrm{e}_{m}\right\rangle_{\pi},
$$

where for each $n, \mathrm{e}_{n}$ is defined by $\mathrm{e}_{n}(k)=\delta_{n, k}$ for $k=1,2, \ldots$ A straightforward computation shows that the 'matrix' $\left(P_{t}(m, n)\right)_{(m, n) \in \mathbb{N}^{*} \times \mathbb{N}^{*}}$ is a solution of the Kolmogorov equation

$$
\frac{\mathrm{d} P_{t}(n, m)}{\mathrm{d} t}=\left(L P_{t}\right)(n, m)=\left(P_{t} L\right)(n, m) .
$$

Furthermore, one can verify that there exists some $M \geq 1$ such that for all $t$ and all $n,\left|\sum_{k=1}^{\infty} P_{t}(n, k)\right| \leq M$. The uniqueness of such a family has been proven in [12, Theorem 14 p. 528] under Assumption (2.12). This implies that the symmetric sub-markovian semigroup $\left(P_{t}, t \geq 0\right)$ is the extension of the transition semigroup of the Markov process $\left(X_{t}^{K}, t \geq 0\right)$ to $\ell^{2}(\pi)$.

In what follows, the solution $u^{0}=\left(u_{n}^{0}\right)_{n \in \mathbb{N}^{*}}$ of the homogeneous equation

$$
\lambda_{n} u_{n+1}+\mu_{n} u_{n-1} \mathbb{1}_{\{n \geq 2\}}-\left(\lambda_{n}+\mu_{n}\right) u_{n}=0
$$

such that $u_{1}^{0}=1$ will play an important role. Using (2.11) it is easy to verify that

$$
u_{n}^{0}=1+\sum_{j=1}^{n-1} \frac{1}{\lambda_{j} \pi_{j}}, n \geq 1
$$

with the convention that $\sum_{j=1}^{0}=0$.

REMARK 3.2. Notice that $u^{0} \notin \ell^{2}(\pi)$. Indeed, using (2.11), observe that

$$
u_{n}^{0} \geq \frac{1}{\lambda_{n-1} \pi_{n-1}}=\frac{1}{\mu_{n} \pi_{n}} .
$$

Hence

$$
\sum_{n=1}^{N}\left(u_{n}^{0}\right)^{2} \pi_{n} \geq \sum_{n=1}^{N} \frac{1}{\mu_{n}^{2} \pi_{n}} .
$$

But by (2.15) the last sum tends to $+\infty$ when $N$ goes to infinity. 


\subsection{Estimates of the largest eigenvalue and of the associated eigenvector}

Our first main result gives the behavior of $\rho_{0}$ and $\varphi$ as functions of $K$ when $K$ gets large. Recall that $x_{*}$ and $n_{*}(K)$ are defined in (2.4) and (2.18), respectively, and that $u^{0}=\left(u_{n}^{0}\right)_{n}$ is the solution of the homogeneous equation (3.2). The function $H$ is defined in (2.8) and recall that $H^{\prime \prime}\left(x_{*}\right)>0$ (see $(2.5)$ ).

THEOREM 3.2 (Estimates of $\rho_{0}$ and $\varphi$ ).

For all $K>1$, we have

$$
\begin{aligned}
\rho_{0}(K)= & \frac{\left(\sqrt{\frac{\lambda_{1}}{\mu_{1}}}-\sqrt{\frac{\mu_{1}}{\lambda_{1}}}\right) \sqrt{K H^{\prime \prime}\left(x_{*}\right)} x_{*} \tilde{\lambda}\left(x_{*}\right)}{\sqrt{2 \pi}} e^{-K \int_{0}^{x_{*}} \log \frac{\tilde{\lambda}(x)}{\tilde{\mu}(x)} \mathrm{d} x} \\
& \times\left(1+\mathcal{O}\left(\frac{(\log K)^{3}}{\sqrt{K}}\right)\right) .
\end{aligned}
$$

Moreover, for all $K>1$, we have

$$
\sup _{n \in \mathbb{N}^{*}}\left|\varphi_{n}(K)-V_{n}(K)\right| \leq \mathcal{O}(1) \rho_{0}(K) K \log K
$$

where

$$
V_{n}(K)= \begin{cases}u_{n}^{0} & \text { if } n \leq n_{*}(K) \\ u_{n_{*}(K)}^{0} & \text { if } n \geq n_{*}(K) .\end{cases}
$$

The proof of this theorem is given in Section [5. Notice that the constant $c$ defined by

$$
c=-\int_{0}^{x_{*}} \log \frac{\tilde{\mu}(x)}{\tilde{\lambda}(x)} \mathrm{d} x
$$

is strictly positive by the assumptions on the functions $\tilde{\lambda}, \tilde{\mu}$. It will appear several times later on.

REMARK 3.3. In the logistic case, one finds

$$
\rho_{0}(K)=\frac{(\tilde{\lambda}-\tilde{\mu})^{2}}{\sqrt{2 \pi \tilde{\mu}}} \sqrt{K} e^{-K\left(\tilde{\lambda}-\tilde{\mu}+\tilde{\mu} \log \frac{\tilde{\mu}}{\lambda}\right)}\left(1+\mathcal{O}\left(\frac{(\log K)^{3}}{\sqrt{K}}\right)\right) .
$$

The following theorem provides a lower bound for the spectral gap.

Theorem 3.3 (Spectral gap).

There exists a constant $d>0$ such that for all $K>1$

$$
\rho_{1}(K)-\rho_{0}(K) \geq \frac{d}{\log K} .
$$

The proof of this theorem is given in Section 6 ,

REMARK 3.4. As a consequence of the preceding two theorems, one has $\rho_{0}(K) \ll \rho_{1}(K)-\rho_{0}(K)$ for large $K$ because $\rho_{0}(K) \approx \sqrt{K} e^{-c K}$. 


\subsection{Quasi-stationary distribution, survival rate and mean time to extinction}

We refer to [18] and [7] for background and more informations about quasistationary distributions. As usual, we shall denote by $\mathbb{P}_{\nu}$ the law of the process starting from a distribution $\nu$ and by $\mathbb{P}_{n}$ the law of the process starting from the state $n$, i.e. starting from the distribution $\delta_{n}$. The corresponding exepectations are respectively denoted by $\mathbb{E}_{\nu}$ and $\mathbb{E}_{n}$.

\section{Proposition 3.4.}

For all $K>1$, the probability measure $\nu=\left(\nu_{n}\right)_{n}$ on $\mathbb{N}^{*}$ defined by

$$
\nu_{n}=\frac{\pi_{n} \varphi_{n}}{\langle\varphi, \mathbb{1}\rangle_{\pi}}
$$

is the unique quasi-stationary distribution of the birth and death process.

Note that the quasi-stationary distribution $\nu$ depends on $K$ through $\varphi$. Proof. In order to prove that $\nu$ is a quasi-stationary distribution, we must verify that $\mathbb{P}_{\nu}\left(X_{t}^{K} \in A \mid T_{0}>t\right)=\nu(A)$ for all $t>0$ and for all subsets $A \subseteq \mathbb{N}^{*}$. Observe that for all $A \subseteq \mathbb{N}^{*}, \mathbb{1}_{A} \in \ell^{2}(\pi)$. We have, using that $L$ is self-adjoint,

$$
\begin{aligned}
\mathbb{P}_{\nu}\left(X_{t}^{K} \in A, T_{0}>t\right) & =\sum_{n \in \mathbb{N}^{*}} \nu_{n} P_{t}(n, A)=\frac{\left\langle\varphi, e^{t L} \mathbb{1}_{A}\right\rangle_{\pi}}{\langle\varphi, \mathbb{1}\rangle_{\pi}} \\
& =\frac{\left\langle e^{t L} \varphi, \mathbb{1}_{A}\right\rangle_{\pi}}{\langle\varphi, \mathbb{1}\rangle_{\pi}}=e^{-\rho_{0} t} \frac{\left\langle\varphi, \mathbb{1}_{A}\right\rangle_{\pi}}{\langle\varphi, \mathbb{1}\rangle_{\pi}} \\
& =e^{-\rho_{0} t} \nu(A) .
\end{aligned}
$$

Replacing $A$ by $\mathbb{N}^{*}$ yields the wanted relation. Since we have uniqueness (by (2.13) $), \nu$ must be the quasi-stationary distribution.

Before proceeding with the other results, we observe that the previous proof shows that for all $t>0$

$$
\mathbb{P}_{\nu}\left(T_{0}>t\right)=e^{-\rho_{0} t} .
$$

The quantity $\rho_{0}$ is usually called the exponential rate of survival. The mean time to extinction (starting from the quasi-stationary distribution) is thus

$$
\mathbb{E}_{\nu}\left[T_{0}\right]=\frac{1}{\rho_{0}(K)} .
$$

In view of Theorem 3.2, it is of order $e^{c K} / \sqrt{K}$ for some positive constant c. More precisely, we have the following corollary. 
Corollary 3.5 (Approximation of the mean time to extinction). For all $K>1$ we have

$$
\begin{aligned}
\mathbb{E}_{\nu}\left[T_{0}\right]= & \frac{\sqrt{2 \pi}}{\left(\sqrt{\frac{\lambda_{1}}{\mu_{1}}}-\sqrt{\frac{\mu_{1}}{\lambda_{1}}}\right) \sqrt{K H^{\prime \prime}\left(x_{*}\right)} x_{*} \tilde{\lambda}\left(x_{*}\right)} e^{K \int_{0}^{x_{*}} \log \frac{\tilde{\lambda}(x)}{\tilde{\mu}(x)} \mathrm{d} x} \\
& \times\left(1+\mathcal{O}\left(\frac{(\log K)^{3}}{\sqrt{K}}\right)\right) .
\end{aligned}
$$

Note that there is another way to obtain the above estimate of $\mathbb{E}_{\nu}\left[T_{0}\right]$. Indeed, we have

$$
\mathbb{E}_{\nu}\left[T_{0}\right]=\sum_{n \in \mathbb{N}^{*}} \mathbb{E}_{n}\left[T_{0}\right] \nu_{n}
$$

and since (see [13])

$$
\mathbb{E}_{n}\left[T_{0}\right]=\sum_{m=1}^{n} \frac{1}{\lambda_{m} \mu_{m}} \sum_{i \geq m+1} \pi_{i},
$$

the estimate can be obtained by using Proposition 3.4 and Theorem 3.2 to deal with $\varphi_{n}$.

\subsection{Convergence rate to the quasi-stationary distribution and Gaussian approximation}

We denote by $\mathrm{d}_{\mathrm{TV}}\left(\mu^{(1)}, \mu^{(2)}\right)$ the total variation distance between two probability measures $\mu^{(1)}$ and $\mu^{(2)}$. Recall that

$$
\mathrm{d}_{\mathrm{TV}}\left(\mu^{(1)}, \mu^{(2)}\right)=\sup _{A \in \mathscr{P}(\mathbb{N})}\left|\mu^{(1)}(A)-\mu^{(2)}(A)\right|=\frac{1}{2} \sum_{n \in \mathbb{N}}\left|\mu_{n}^{(1)}-\mu_{n}^{(2)}\right|
$$

where $\mathscr{P}(\mathbb{N})$ is the powerset of $\mathbb{N}$.

The process $\left(X_{t}^{K}, t \geq 0\right)$ is said to have a Yaglom limit if there exists a probability measure $\mathfrak{m}$ on $\mathbb{N}^{*}$ such that for every $n \in \mathbb{N}^{*}$ and for every $A \in \mathscr{P}\left(\mathbb{N}^{*}\right)$ one has

$$
\lim _{t \rightarrow \infty} \mathbb{P}_{n}\left(X_{t}^{K} \in A \mid T_{0}>t\right)=\mathfrak{m}(A) .
$$

When it exists, the Yaglom limit is a quasi-stationary distribution (whereas the converse is false in general), see [18].

The following theorem provides a quantitative bound for the distance (in total variation) between the law of the process and a convex combination of the Dirac mass at 0 and the quasi-stationary distribution $\nu$. It also shows that $\nu$ is the Yaglom limit of $\left(X_{t}^{K}, t \geq 0\right)$ with a quantitative error bound. Recall that $-\rho_{1}$ is the second largest eigenvalue of $L$ (see Theorem 3.1). 


\section{THEOREM 3.6.}

There exist three strictly positive constants a, $c_{1}, C$ such that for all $K>1$, for all $n \in \mathbb{N}^{*}$ and for all $t \geq 0$, we have

$$
\begin{aligned}
& \mathrm{d}_{\mathrm{TV}}\left(\mathbb{P}_{n}\left(X_{t}^{K} \in \cdot\right), \alpha_{n}(K) \nu+\left(1-\alpha_{n}(K)\right) \delta_{0}\right) \\
& \leq C\left(K^{3 / 2} \log K e^{-c K}+\left(1-e^{-\rho_{0} t}\right)+K e^{-\frac{a}{4} t}+K^{\frac{3}{4}} e^{c_{1} K} e^{-\frac{\rho_{1}}{2} t}\right)
\end{aligned}
$$

where

$$
\alpha_{n}(K)= \begin{cases}\frac{u_{n}^{0}}{u_{n^{*}(K)}} & \text { for } n \leq n_{*}(K) \\ 1 & \text { for } n \geq n_{*}(K)\end{cases}
$$

and where $u^{0}$ is defined in (3.3). Moreover

$$
\mathrm{d}_{\mathrm{TV}}\left(\frac{P_{t}(n, \cdot)}{P_{t}\left(n, \mathbb{N}^{*}\right)}, \nu\right) \leq C\left(K e^{-\left(\frac{a}{4}-\rho_{0}\right) t}+K^{\frac{3}{4}} e^{c_{1} K} e^{-\left(\frac{\rho_{1}}{2}-\rho_{0}\right) t}\right) .
$$

In particular, the probability measure $\nu$ is the Yaglom limit (in total variation distance) of the process $\left(X_{t}^{K}, t \geq 0\right)$.

The proof of this theorem is given in Section 7

REMARK 3.5. The proof of the previous theorem consists in establishing the following more explicit estimate: there exist three strictly positive constants $a, c_{1}, C$ such that for all $K>1$, for all $n \in \mathbb{N}^{*}$ and for all $t \geq 0$, we have

$$
\begin{aligned}
& \mathrm{d}_{\mathrm{TV}}\left(\mathbb{P}_{n}\left(X_{t}^{K} \in \cdot\right), \frac{\langle\varphi, \mathbb{1}\rangle_{\pi}}{\|\varphi\|_{\pi}^{2}} e^{-\rho_{0} t} \varphi_{n} \nu(\cdot)+\left(1-\frac{\langle\varphi, \mathbb{1}\rangle_{\pi}}{\|\varphi\|_{\pi}^{2}} e^{-\rho_{0} t} \varphi_{n}\right) \delta_{0}(\cdot)\right) \\
& \leq C\left(K e^{-\frac{a}{4} t}+K^{\frac{3}{4}} e^{c_{1} K} e^{-\frac{\rho_{1}}{2} t}\right) .
\end{aligned}
$$

Then we show that the estimates (3.5) and (3.6) follow from (3.7).

REMARK 3.6. The estimate (3.5) can be interpreted as follows. Recall that, for $K$ large, $\rho_{0}$ is very small. Therefore, if we start with $n=\mathcal{O}(K)$ and if $t$ is such that $K \log K /\left(\rho_{1}-\rho_{0}\right) \ll t \ll 1 / \rho_{0}$, we get the following rough estimate:

$$
\mathrm{d}_{\mathrm{TV}}\left(\mathbb{P}_{n}\left(X_{t}^{K} \in \cdot\right), \alpha_{n}(K) \nu+\left(1-\alpha_{n}(K)\right) \delta_{0}\right) \ll 1 .
$$

This inequality highlights the existence of an interval of time during which the process is either extinct with a probability close to $1-\alpha_{n}(K)$ or obeys the quasi-stationary distribution $\nu$ with a probability close $\alpha_{n}(K)$. This interval has a length that is roughly exponentially large in $K$.

REMARK 3.7. It follows from Theorem 3.2 and Theorem 3.3 that, for $K$ large enough,

$$
\min \left(\frac{a}{4}-\rho_{0}, \frac{\rho_{1}}{2}-\rho_{0}\right) \geq \frac{d}{3 \log K} .
$$


Hence, for $K$ large enough, the estimate (3.6) can be written as

$$
\mathrm{d}_{\mathrm{TV}}\left(\frac{P_{t}(n, \cdot)}{P_{t}\left(n, \mathbb{N}^{*}\right)}, \nu\right) \leq 2 C K e^{c_{1} K} e^{-\frac{d}{3 \log K} t} .
$$

REMARK 3.8. Note that for every $n \geq 1$, the weights $\alpha_{n}(K)$ appearing in (3.5) can be written as

$$
\alpha_{n}(K)=1-\left(\frac{\mu_{1}}{\lambda_{1}}\right)^{n}+\frac{\mathcal{O}(1)}{K}
$$

for all $K>1$. This follows by adapting the proof of Lemma 9.5 .

The last result shows that the quasi-stationary distribution $\nu$ is close, as $K$ gets large, to a Gaussian law centered at $n_{*}(K)$. Recall that the function $H$ is defined in (2.8).

\section{THEOREM 3.7.}

We have

$$
\mathrm{d}_{\mathrm{TV}}\left(\nu^{K}, G^{K}\right) \leq \frac{\mathcal{O}(1)}{\sqrt{K}}
$$

where $G^{K}$ is the probability measure on $\mathbb{N}^{*}$ given by

$$
G_{n}^{K}=\frac{1}{Z(K)} e^{-\frac{\left(n-n_{*}(K)\right)^{2}}{2 K \sigma^{2}}}
$$

where

$$
Z(K)=\sum_{n=1}^{\infty} e^{-\frac{\left(n-n_{*}(K)\right)^{2}}{2 K \sigma^{2}}}=\sqrt{2 \pi K} \sigma+\mathcal{O}(1)
$$

and where

$$
\sigma=\frac{1}{\sqrt{H^{\prime \prime}\left(x_{*}\right)}}
$$

Recall that $H^{\prime \prime}\left(x_{*}\right)>0$ by (2.17). In the logistic case, one has $\sigma=\sqrt{\tilde{\lambda}}$. The proof of this theorem is given in Section 8 .

\section{Proof of Theorem 3.1}

\section{1 $\tilde{L}$ is symmetric and closable in $\ell^{2}(\pi)$}

Using (2.11), the reader can verify that, for all $u, v \in \mathscr{D}$, one has $\langle\tilde{L} u, v\rangle_{\pi}=$ $\langle u, \tilde{L} v\rangle_{\pi}$. Hence $\tilde{L}$ is symmetric.

To verify closedness, one can apply a result in [14, III.5.3] saying that it is equivalent to prove that, for every sequence $\left(y^{(k)}\right)_{k} \in \mathscr{D}$ such that $y^{(k)} \rightarrow 0$ (in $\ell^{2}(\pi)$ ) and such that $\tilde{L} y^{(k)}$ converges to $y$ (in $\left.\ell^{2}(\pi)\right), y=0$. Details are left to the reader. 


\section{2 $L$ defines a positive contraction semigroup in $\ell^{2}(\pi)$}

The key result in proving this claim is the following.

\section{Proposition 4.1.}

For every $f \in \ell^{2}(\pi)$ and every $\rho>0$, the equation

$$
(\rho-L) y=f
$$

has a unique solution $y \in \mathcal{D}$ denoted by $R_{\rho} f$. Moreover

$$
\left\|R_{\rho} f\right\|_{\pi} \leq \rho^{-1}\|f\|_{\pi} .
$$

Finally, if $f$ is nonnegative, so is $R_{\rho} f$.

It is well-known that the previous bound is a sufficient condition for $L$ to generate a $C_{0}$ contraction semigroup $Q_{t}$ in $\ell^{2}(\pi)$, see e.g. [24, p. 249].

The proof of this proposition requires two preliminary results. For $1 \leq$ $n \leq N$ we define (on $\ell^{\infty}(\{1, \ldots, N\})$ ) the truncated operator $L_{N}$ by

$$
\left(L_{N} v\right)_{n}=\lambda_{n} v_{n+1} \mathbb{1}_{\{n<N\}}+\mu_{n} v_{n-1} \mathbb{1}_{\{n \geq 2\}}-\left(\lambda_{n}+\mu_{n}\right) v_{n} .
$$

The operator $L_{N}$ satisfies the following positive maximum principle.

LEMMA 4.2.

Let $v \in \ell^{\infty}(\{1, \ldots, N\})$ and let $m \in\{1, \ldots, N\}$ such that $v_{m}=\sup _{1 \leq n \leq N} v_{n}$.

If $v_{m} \geq 0$, then $\left(L_{N} v\right)_{m} \leq 0$.

Proof. For $2 \leq m \leq N-1$, we get

$$
\left(L_{N} v\right)_{m}=\lambda_{m} v_{m+1}+\mu_{m} v_{m-1}-\left(\lambda_{m}+\mu_{m}\right) v_{m} \leq 0
$$

since, by definition of $m, v_{m}$ is maximal. The cases $m=1$ and $m=N$ follow similarly.

\section{LEMMA 4.3.}

Let $g \in \ell^{\infty}(\{1, \ldots, N\})$ and $\rho>0$. The equation $\left(\rho-L_{N}\right) v=g$ has a unique

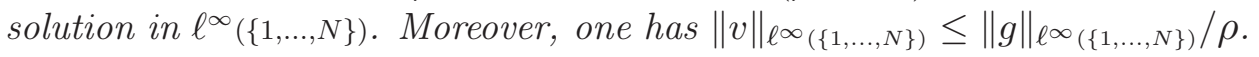
Finally, if $g \geq 0$ then $v \geq 0$.

Proof. If $g \in \ell^{\infty}(\{1, \ldots, N\})$ and $\rho>0$ are such that $g=\left(\rho-L_{N}\right) v$, and if $m \in\{1, \ldots, N\}$ is such that $v_{m}=\sup _{1<n<N} v_{n} \geq 0$ then, by Lemma 4.2. $v_{m} \leq g_{m} / \rho$. Considering $-v$ and $-g$, it follows that if $\left(\rho-L_{N}\right) v=g$ and if $m \in\{1, \ldots, N\}$ is such that $v_{m}=\inf _{1 \leq n \leq N} v_{n} \leq 0$ then $v_{m} \geq g_{m} / \rho$. This implies that $v \geq 0$ if $g \geq 0$. The previous two inequalities imply $\|v\|_{\ell^{\infty}(\{1, \ldots, N\})} \leq\|g\|_{\ell^{\infty}(\{1, \ldots, N\})} / \rho$. In particular we have $\operatorname{Ker}\left(\rho-L_{N}\right)=\{0\}$, namely $\rho-L_{N}$ is invertible in $\ell^{\infty}(\{1, \ldots, N\})$. The lemma is proved. 
We now turn to the proof of Proposition 4.1.

Let $f \in \mathscr{D}$ and let $N_{0} \geq 1$ be such that $f_{n}=0$ for all $n>N_{0}$. Applying Lemma 4.3 for $N>N_{0}$ yields a $v^{(N)} \in \ell^{\infty}(\{1, \ldots, N\})$ such that

$$
\left(\rho-L_{N}\right) v^{(N)}=f .
$$

We also have that for all $N>N_{0}$

$$
\left\|v^{(N)}\right\|_{\ell^{\infty}(\{1, \ldots, N\})} \leq \frac{1}{\rho}\|f\|_{\ell^{\infty}\left(\left\{1, \ldots, N_{0}\right\}\right)} .
$$

Define $u^{(N)} \in \mathscr{D}$ by

$$
u_{n}^{(N)}=\left\{\begin{array}{lll}
v_{n}^{(N)} & \text { if } & n \leq N \\
0 & \text { if } & n>N
\end{array}\right.
$$

For all $p \in \mathbb{N}^{*}$ we have

$$
\left((\rho-L) u^{(N)}\right)_{p}=f_{p}+\left[\left(\rho+\lambda_{N}+\mu_{N}\right) v_{N}^{(N)}-\mu_{N} v_{N-1}^{(N)}\right] \mathbb{1}_{\{p=N\}}-\mu_{N+1} v_{N}^{(N)} \mathbb{1}_{\{p=N+1\}} .
$$

It is then easy to show that

$$
\left\|(\rho-L) u^{(N)}-f\right\|_{\pi}^{2} \leq \mathcal{O}(1)\left(\pi_{N} \mu_{N}^{2}+\pi_{N+1} \mu_{N+1}^{2}\right)
$$

by using (2.2) and (4.1). Hence, since we assume that (2.15) holds, we get that $(\rho-L) u^{(N)}$ converges strongly to $f$. Using $u_{N+1}^{(N)}=0$ we obtain

$$
\left\langle u^{(N)},(\rho-L) u^{(N)}\right\rangle_{\pi}=\left\langle u^{(N)}, f\right\rangle_{\pi}+r_{N}
$$

where $r_{N}=\left[\left(\rho+\lambda_{N}+\mu_{N}\right) v_{N}^{(N)}-\mu_{N} v_{N-1}^{(N)}\right] v_{N}^{(N)} \pi_{N}$.

One gets (recall that $u_{N+1}^{(N)}=0$ )

$$
\begin{aligned}
\left\langle u^{(N)}, L u^{(N)}\right\rangle_{\pi} & \sum_{n=1}^{\infty} \pi_{n} \lambda_{n} u_{n}^{(N)} u_{n+1}^{(N)}+\sum_{n=2}^{\infty} \pi_{n} \mu_{n} u_{n}^{(N)} u_{n-1}^{(N)}-\sum_{n=1}^{\infty} \pi_{n}\left(\lambda_{n}+\mu_{n}\right)\left(u_{n}^{(N)}\right)^{2} \\
\leq & \frac{1}{2} \sum_{n=1}^{\infty} \pi_{n} \lambda_{n}\left(u_{n}^{(N)}\right)^{2}+\frac{1}{2} \sum_{n=1}^{\infty} \pi_{n} \lambda_{n}\left(u_{n+1}^{(N)}\right)^{2}+\frac{1}{2} \sum_{n=2}^{\infty} \pi_{n} \mu_{n}\left(u_{n}^{(N)}\right)^{2} \\
& +\frac{1}{2} \sum_{n=2}^{\infty} \pi_{n} \mu_{n}\left(u_{n-1}^{(N)}\right)^{2}-\sum_{n=1}^{\infty} \pi_{n}\left(\lambda_{n}+\mu_{n}\right)\left(u_{n}^{(N)}\right)^{2} \\
\leq & \frac{1}{2} \sum_{n=1}^{\infty} \pi_{n} \lambda_{n}\left(u_{n}^{(N)}\right)^{2}+\frac{1}{2} \sum_{n=1}^{\infty} \pi_{n+1} \mu_{n+1}\left(u_{n+1}^{(N)}\right)^{2}+\frac{1}{2} \sum_{n=2}^{\infty} \pi_{n} \mu_{n}\left(u_{n}^{(N)}\right)^{2} \\
& +\frac{1}{2} \sum_{n=1}^{\infty} \pi_{n} \lambda_{n}\left(u_{n}^{(N)}\right)^{2}-\sum_{n=1}^{\infty} \pi_{n}\left(\lambda_{n}+\mu_{n}\right)\left(u_{n}^{(N)}\right)^{2} \\
\leq & -\pi_{1} \mu_{1}\left(u_{1}^{(N)}\right)^{2} \leq 0
\end{aligned}
$$


where we used (2.11). Hence, it follows from (4.2) and the previous inequality that

$$
\begin{aligned}
\rho\left\|u^{(N)}\right\|_{\pi}^{2} & =\left\langle u^{(N)},(\rho-L) u^{(N)}\right\rangle_{\pi}+\left\langle u^{(N)}, L u^{(N)}\right\rangle_{\pi} \\
& \leq\left\langle u^{(N)}, f\right\rangle_{\pi}+r_{N} \leq\left\|u^{(N)}\right\|_{\pi}\|f\|_{\pi}+r_{N} .
\end{aligned}
$$

Therefore we obtain

$$
\left\|u^{(N)}\right\|_{\pi} \leq \frac{\|f\|_{\pi}}{2 \rho}+\sqrt{\frac{r_{N}}{\rho}+\frac{\|f\|_{\pi}^{2}}{4 \rho^{2}}}
$$

where the right hand side is the largest root of the polynomial function $x \mapsto \rho x^{2}-\|f\|_{\pi} x-r_{N}$. Since $r_{N}$ tends to 0 by (2.15) when $N$ tends to infinity, $\sup _{N}\left\|u^{(N)}\right\|_{\pi}<\infty$. Since a ball in the Hilbert space $\ell^{2}(\pi)$ is weakly compact [24, p. 126], we can extract from the sequence $\left(u^{(N)}\right)$ a subsequence weakly converging to some $u \in \ell^{2}(\pi)$. Moreover

$$
\|u\|_{\pi} \leq \frac{1}{\rho}\|f\|_{\pi}
$$

by [24, Theorem 1, p. 120]. Since the sequence $\left((\rho-L) u^{(N)}\right)$ is also weakly convergent to $f$ (see above, even strongly convergent in our case), we can apply [14, Problem 5.12, p. 165] to conclude that $u \in \mathcal{D}$ and $(\rho-L) u=f$. At this point, we have proved that for all $f \in \mathscr{D}$ the equation $(\rho-L) u=f$ has a solution in $\mathcal{D}$.

If $f$ is nonnegative, Lemma 4.3 implies that all the $u^{(N)}$ are nonnegative for $N$ large enough, hence $u$ is nonnegative.

For every $w \in \mathcal{D}$, there is a sequence $\left(w^{(n)}\right)$, with $w^{(n)} \in \mathscr{D}$ for all $n$, converging to $w$ (in $\ell^{2}(\pi)$ ) with $\left(L w^{(n)}\right)$ converging to $L w$ in $\ell^{2}(\pi)$ (see [14, III.5.2]). As before,

$$
\left\langle w^{(n)},(\rho-L) w^{(n)}\right\rangle_{\pi} \geq \rho\left\|w^{(n)}\right\|_{\pi}^{2} .
$$

Therefore

$$
\langle w,(\rho-L) w\rangle_{\pi} \geq \rho\|w\|_{\pi}^{2}
$$

for all $w \in \mathcal{D}$. This implies that the equation

$$
(\rho-L) u=f .
$$

has a unique solution $u \in \mathcal{D}$ for every $f \in \mathscr{D}$. This solution, denoted by $R_{\rho} f$, satisfies

$$
\left\|R_{\rho} f\right\|_{\pi} \leq \frac{1}{\rho}\|f\|_{\pi}
$$

and it is nonnegative if $f$ is nonnegative. Since $\mathscr{D}$ is dense in $\ell^{2}(\pi)$, the linear operator $R_{\rho}$ can be extended to a linear operator on $\ell^{2}(\pi)$ with a norm that is at most $\rho^{-1}$ (see [14, II.2.2]). 
Since $\mathscr{D}$ is dense in $\ell^{2}(\pi)$, for each $f \in \ell^{2}(\pi)$ we can find a sequence $\left(f^{(k)}\right) \subset \mathscr{D}$ converging to $f$ in $\ell^{2}(\pi)$. Moreover, $\left(R_{\rho} f^{(k)}\right)$ converges to $R_{\rho} f$. Since, for all $k, R_{\rho} f^{(k)} \in \mathcal{D}$ and $L R_{\rho} f^{(k)}=\rho R_{\rho} f^{(k)}-f^{(k)}$ converges in $\ell^{2}(\pi)$ to $\rho R_{\rho} f-f$, we conclude, by using [14, III.5.2], that, for every $f \in \ell^{2}(\pi)$, $R_{\rho} f \in \mathcal{D}$ and

$$
(\rho-L) R_{\rho} f=f .
$$

Nonnegativity follows easily. This finishes the proof of the proposition.

We can now make the proof of statement 2 in Theorem 3.1. Using Proposition 4.1, we can apply [24, p. 249] to show that $L$ generates a $C_{0}$ contraction semigroup $Q_{t}$ in $\ell^{2}(\pi)$. For all $t \geq 0$, the operator $Q_{t}$ maps nonnegative sequences to nonnegative sequences since this holds for $R_{\rho}$ for all $\rho>0$ using [24, formula 3, p. 246].

\subsection{Compactness, self-adjointness and dissipativity}

$L$ has a compact resolvent in $\ell^{2}(\pi)$. From the equation $(\rho-L) R_{\rho}=\mathrm{Id}$, we get for every $f \in \ell^{2}(\pi)$

$$
\left(R_{\rho} f\right)_{n}=-\frac{f_{n}}{\rho+\lambda_{n}+\mu_{n}}+\frac{\lambda_{n}\left(R_{\rho} f\right)_{n+1}}{\lambda_{n}+\mu_{n}+\rho}+\frac{\mu_{n}\left(R_{\rho} f\right)_{n-1}}{\lambda_{n}+\mu_{n}+\rho} \mathbb{1}_{\{n \geq 2\}} .
$$

We are going to verify that each term is uniformly square summable at infinity with respect to the weights $\left(\pi_{n}\right)$.

This is obvious for the first term since $\lim _{n \rightarrow \infty} \frac{1}{\lambda_{n}+\mu_{n}+\rho}=0$.

For the other two terms, by using (2.11), we have for all $N \geq 2$

$$
\begin{aligned}
& \sum_{n=N}^{\infty}\left|\left(R_{\rho} f\right)_{n+1}\right|^{2} \frac{\lambda_{n}^{2} \pi_{n}}{\left(\lambda_{n}+\mu_{n}+\rho\right)^{2}}+\sum_{n=N}^{\infty}\left|\left(R_{\rho} f\right)_{n-1}\right|^{2} \frac{\mu_{n}^{2} \pi_{n}}{\left(\lambda_{n}+\mu_{n}+\rho\right)^{2}}= \\
& \sum_{n=N}^{\infty}\left|\left(R_{\rho} f\right)_{n+1}\right|^{2} \frac{\lambda_{n} \mu_{n+1} \pi_{n+1}}{\left(\lambda_{n}+\mu_{n}+\rho\right)^{2}}+\sum_{n=N}^{\infty}\left|\left(R_{\rho} f\right)_{n-1}\right|^{2} \frac{\mu_{n} \lambda_{n-1} \pi_{n-1}}{\left(\lambda_{n}+\mu_{n}+\rho\right)^{2}} .
\end{aligned}
$$

Using (2.2) and (2.14) we conclude that for all $\varepsilon>0$, there exists $N_{\varepsilon}$ such that for all $N \geq N_{\varepsilon}$

$$
\begin{aligned}
& \sum_{n=N}^{\infty}\left(\left|\left(R_{\rho} f\right)_{n+1}\right|^{2} \frac{\lambda_{n}^{2} \pi_{n}}{\left(\lambda_{n}+\mu_{n}+\rho\right)^{2}}+\left|\left(R_{\rho} f\right)_{n-1}\right|^{2} \frac{\mu_{n}^{2} \pi_{n}}{\left(\lambda_{n}+\mu_{n}+\rho\right)^{2}}\right) \\
& \leq \varepsilon\left\|R_{\rho} f\right\|_{\pi}^{2} \leq \frac{\varepsilon\|f\|_{\pi}^{2}}{\rho^{2}} .
\end{aligned}
$$

Compactness of the resolvent follows. 
If $-\rho$ is an eigenvalue, a corresponding eigenvector $u$ (in $\left.\ell^{2}(\pi)\right)$ must satisfy the identities

$$
\begin{aligned}
& u_{2}=\frac{\left(\lambda_{1}+\mu_{1}-\rho\right) u_{1}}{\lambda_{1}}, \\
& u_{n+1}=\frac{\left(\lambda_{n}+\mu_{n}-\rho\right) u_{n}}{\lambda_{n}}-\frac{\mu_{n} u_{n-1}}{\lambda_{n}}, \quad \forall n \geq 2 .
\end{aligned}
$$

Therefore, $u_{1}$ determines all the $u_{n}{ }^{\prime} s$. This implies that all eigenvalues are simple.

Positivity of the eigenvector associated with the maximal eigenvalue $-\rho_{0}$ follows from the fact that the semigroup preserves nonnegativity and the fact that if an eigenvector is orthogonal to any positive function, it would be equal to 0 , which is not true.

Self-adjointness and dissipativity. Self-adjointness follows by an argument found in [14, problem V.3.32, p. 279]. In more details, it follows from equation (4.3) that for all $u \in \mathcal{D},\langle u, L u\rangle_{\pi} \leq 0$, hence is $L$ is dissipative and the numerical range of $L$ is contained in the negative real line. By Theorem V.3.2 page 268 in 14 the defect index is constant outside the negative real line, and equal to zero on the positive real line by Proposition 4.1. Therefore the spectrum of $L$ is contained in the negative real line and $L$ is self adjoint by Theorem 3.16 in [14, Chapter V, p. 271].

\section{$5 \quad$ Proof of Theorem 3.2}

For every small number $\rho$, we are going to consider sequences $\left(u_{n}\right)_{n}$ satisfying

$$
\lambda_{n} u_{n+1}+\mu_{n} u_{n-1} \mathbb{1}_{\{n \geq 2\}}-\left(\lambda_{n}+\mu_{n}\right) u_{n}=-\rho u_{n} .
$$

The strategy will be as follows. If $\rho=0, u^{0}$ is a solution of (5.1) for all $n \geq 1$ and the constant sequence 1 is a solution of (5.1) for all $n \geq 2$. For small $\rho \neq 0$ and $n \leq n_{*}(K)$, we will look for a solution of (5.1) that is a small perturbation of $u^{0}$. Since $u^{0} \notin \ell^{2}(\pi)$ (see Remark 3.2), we cannot use such an argument for large $n$. For $n \geq n_{*}(K)-1$, we will use Levinson's technique (see [17, [11) to prove that there is a solution of (5.1) that is almost constant. Then we will match these two solutions in $\left\{n_{*}(K)-1, n_{*}(K)\right\}$. This will be possible for a single value of $\rho$ that has to be $\rho_{0}$. Since (5.1) is a recursion of order 2 , this matched sequence is a solution for all $n \in \mathbb{N}^{*}$. Finally we will prove that this sequence belongs to $\mathcal{D}$ (see Theorem 3.1 for the definition of $\mathcal{D})$.

\subsection{When $1 \leq n \leq n_{*}(K)$}

We look for a solution of the form

$$
v_{n}=u_{n}^{0}\left(1+\delta_{n}\right)
$$


where $u^{0}=\left(u_{n}^{0}\right)$ is defined in (3.3).

\section{Proposition 5.1.}

There exists a constant $\widetilde{C}>0$ such that for $K$ large enough and for each $\rho \in[-1 /(3 \widetilde{C} K \log K), 1 /(3 \widetilde{C} K \log K)]$ the equation (5.1) admits for all $n \leq n_{*}(K)$ a solution of the form

$$
v_{n}=u_{n}^{0}\left(1+\delta_{n}\right)
$$

where

1. $\delta_{1}=0$;

2. $\delta_{n}$ is a solution of

$$
\lambda_{n} \frac{u_{n+1}^{0}}{u_{n}^{0}}\left(\delta_{n+1}-\delta_{n}\right)-\mu_{n} \frac{u_{n-1}^{0}}{u_{n}^{0}}\left(\delta_{n}-\delta_{n-1}\right) \mathbb{1}_{\{n \geq 2\}}=-\rho\left(1+\delta_{n}\right) ;
$$

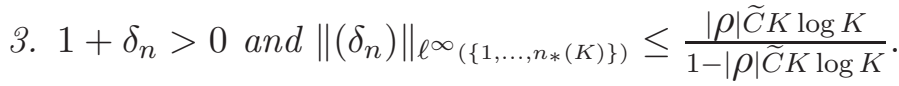

4. $\delta=\left(\delta_{n}\right)_{n}$ is a smooth function of $\rho$ and

$$
\left\|\frac{\mathrm{d} \delta}{\mathrm{d} \rho}(\rho)-\Delta^{0}\right\|_{\ell_{\left(\left\{1, \ldots, n_{*}(K)\right\}\right)}} \leq 4(\widetilde{C} K \log K)^{2}|\rho|
$$

where

$$
\Delta_{n}^{0}=\sum_{j=1}^{n-1} \frac{1}{\lambda_{j} \pi_{j} u_{j}^{0} u_{j+1}^{0}}+\sum_{j=1}^{n-1} \sum_{p=2}^{j} \frac{\left(u_{p}^{0}\right)^{2} \pi_{p}}{\lambda_{j} \pi_{j} u_{j}^{0} u_{j+1}^{0}} \quad \text { for all } n \geq 2
$$

and $\Delta_{1}^{0}=0$.

Proof. It is easy to check that

$$
\lambda_{n} \frac{u_{n+1}^{0}}{u_{n}^{0}}\left(\delta_{n+1}-\delta_{n}\right)-\mu_{n} \frac{u_{n-1}^{0}}{u_{n}^{0}}\left(\delta_{n}-\delta_{n-1}\right) \mathbb{1}_{\{n \geq 2\}}=-\rho\left(1+\delta_{n}\right) .
$$

We impose $\delta_{1}=0$ (i.e. $v_{1}=1$ ).

We now apply Lemma 9.7 for $n \geq 2$ with

$$
h_{n}=-\rho\left(1+\delta_{n}\right), \quad \alpha_{n}=\lambda_{n} \frac{u_{n+1}^{0}}{u_{n}^{0}}, \quad \beta_{n}=\mu_{n} \frac{u_{n-1}^{0}}{u_{n}^{0}} .
$$

For $r>s$, we have

$$
\Theta_{r, s}=\frac{\beta_{r-1} \ldots \beta_{s}}{\alpha_{r-1} \ldots \alpha_{s}}=\frac{\mu_{r-1} \ldots \mu_{s}}{\lambda_{r-1} \ldots \lambda_{s}} \frac{u_{s-1}^{0}}{u_{r-1}^{0}} \frac{u_{s}^{0}}{u_{r}^{0}}=\frac{\lambda_{s-1} \pi_{s-1}}{\lambda_{r-1} \pi_{r-1}} \frac{u_{s-1}^{0}}{u_{r-1}^{0}} \frac{u_{s}^{0}}{u_{r}^{0}} .
$$


Observing that $\lambda_{1} u_{2}^{0} \delta_{2}=-\rho$, we get

$$
\delta_{n}=-\rho\left(\sum_{j=1}^{n-1} \frac{1}{\lambda_{j} \pi_{j} u_{j}^{0} u_{j+1}^{0}}+\sum_{j=1}^{n-1} \sum_{p=2}^{j} \frac{\left(u_{p}^{0}\right)^{2} \pi_{p}\left(1+\delta_{p}\right)}{\lambda_{j} \pi_{j} u_{j}^{0} u_{j+1}^{0}}\right) .
$$

Equation (5.3) can be written as

$$
\delta=-\rho \Delta^{0}+\rho B \delta
$$

where $B$ is a linear operator defined as

$$
(B \delta)_{n}=-\sum_{j=1}^{n-1} \sum_{p=2}^{j} \frac{\left(u_{p}^{0}\right)^{2} \pi_{p}}{\lambda_{j} \pi_{j} u_{j}^{0} u_{j+1}^{0}} \delta_{p} .
$$

Using Lemma 9.3 and the fact that $\mu_{\ell} / \lambda_{\ell}<1$ for $\ell \leq n_{*}(K)-1$, we have the bound

$$
\begin{aligned}
\Delta_{n_{*}(K)}^{0} & =\sum_{j=1}^{n_{*}(K)-1} \frac{1}{\lambda_{j} \pi_{j} u_{j}^{0} u_{j+1}^{0}}+\sum_{j=1}^{n_{*}(K)-1} \sum_{p=2}^{j} \frac{\left(u_{p}^{0}\right)^{2} \pi_{p}}{\lambda_{j} \pi_{j} u_{j}^{0} u_{j+1}^{0}} \\
& \leq \mathcal{O}(1)\left(\sum_{j=1}^{n_{*}(K)-1} \frac{1}{\lambda_{j} \pi_{j}}+\sum_{j=1}^{n_{*}(K)-1} \sum_{p=2}^{j} \frac{1}{\lambda_{p}}\right) \\
& \leq \widetilde{C} K \log K
\end{aligned}
$$

where $\widetilde{C}>0$ is a constant independent of $K$ since $\lambda_{p} \geq p \tilde{\lambda}(0)$. Therefore

$$
\begin{aligned}
& \left\|\Delta^{0}\right\|_{\ell^{\infty}\left(\left\{1, \ldots, n_{*}(K)\right\}\right)} \leq \widetilde{C} K \log K \\
& \text { and }\|B\|_{\ell^{\infty}\left(\left\{1, \ldots, n_{*}(K)\right\}\right)} \leq \Delta_{n_{*}(K)}^{0} \leq \widetilde{C} K \log K .
\end{aligned}
$$

We denote by $\Omega$ the complex disk centered at the origin and of radius $\frac{1}{3 \widetilde{C} K \log K}$. For every $\rho \in \Omega$, the operator Id $-\rho B$ is invertible and $\delta=$ (Id $-\rho B)^{-1} \rho \Delta^{0}$. It follows from (5.4) that

$$
\|\delta\|_{\ell^{\infty}\left(\left\{1, \ldots, n_{*}(K)\right\}\right)} \leq \frac{|\rho| \widetilde{C} K \log K}{1-|\rho| \widetilde{C} K \log K} .
$$

Therefore, $\delta$ is bounded in $\ell^{\infty}\left(\left\{1, \ldots, n_{*}(K)\right\}\right)$ by $\frac{1}{2}$ and $1+\delta_{n}>0$ for all $n \leq$ $n_{*}(K)$. It also follows that $\delta=\left(\delta_{n}\right)_{1 \leq n_{*}(K)}$ is an analytic function on $\Omega$. We now compute its derivative in $\Omega$ :

$$
\frac{\mathrm{d} \delta}{\mathrm{d} \rho}(\rho)=(\mathrm{Id}-\rho B)^{-1} \Delta^{0}+(\mathrm{Id}-\rho B)^{-2} \rho B \Delta^{0} .
$$

Using (5.4) we get that for every $\rho \in \Omega$

$$
\left\|\frac{\mathrm{d} \delta}{\mathrm{d} \rho}(\rho)-\Delta^{0}\right\|_{\ell^{\infty}\left(\left\{1, \ldots, n_{*}(K)\right\}\right)} \leq 4(\widetilde{C} K \log K)^{2}|\rho| .
$$

This finishes the proof of the proposition. 


\subsection{When $n \geq n_{*}(K)-1$}

\section{Proposition 5.2.}

Let $C$ be the constant defined in Lemma 9.1. For $K$ large enough and each $\rho \in[-1 /(3 C K), 1 /(3 C K)]$ the equation (5.1) admits for all $n \geq n_{*}(K)-1$ a solution

$$
v_{n}=1+w_{n},
$$

where

1. $w_{n_{*}(K)-1}=0$;

2. $w_{n}$ is a solution of $\lambda_{n}\left(w_{n+1}-w_{n}\right)+\mu_{n}\left(w_{n-1}-w_{n}\right)=-\rho\left(1+w_{n}\right)$;

3. $1+w_{n}>0$ and $\left\|w_{n}\right\|_{\ell^{\infty}\left(\left\{n_{*}(K)-1, n_{*}(K), \ldots\right\}\right)} \leq \frac{|\rho| C K}{1-|\rho| C K}$.

4. $w=\left(w_{n}\right)$ is a smooth function of $\rho$ and

$$
\left\|\frac{\mathrm{d} w}{\mathrm{~d} \rho}(\rho)-W^{0}\right\|_{\ell^{\infty}\left(\left\{n_{*}(K)-1, n_{*}(K), \ldots\right\}\right)} \leq 4(C K)^{2}|\rho|
$$

where

$$
W_{n}^{0}=\sum_{j=n_{*}(K)}^{n-1} \sum_{p=j+1}^{\infty} \frac{\pi_{p}}{\lambda_{j} \pi_{j}} \quad \text { for all } n \geq n_{*}(K)
$$

and $W_{n_{*}(K)-1}=0$.

Proof. Let us define by induction for $n \geq n_{*}(K)$,

$$
w_{n}=\rho \sum_{j=n_{*}(K)}^{n-1} \sum_{p=j+1}^{\infty} \frac{\pi_{p}}{\lambda_{j} \pi_{j}}\left(1+w_{j}\right),
$$

with $w_{n_{*}(K)-1}=0$. It is easy to check by using (2.11) that

$$
\lambda_{n} w_{n+1}+\mu_{n} w_{n-1}-\left(\lambda_{n}+\mu_{n}\right) w_{n}=-\rho\left(1+w_{n}\right) .
$$

Equation (5.6) can be written as

$$
w=\rho W^{0}+\rho A w
$$

where $A$ is a linear operator defined as

$$
(A w)_{n}=\sum_{j=n_{*}(K)}^{n-1} \sum_{p=j+1}^{\infty} \frac{\pi_{p}}{\lambda_{j} \pi_{j}} w_{j} .
$$

The second assertion in Lemma 9.1 yields the following estimates:

$$
\left\|W^{0}\right\|_{\ell^{\infty}\left(\left\{n_{*}(K)-1, n_{*}(K), \ldots\right\}\right)} \leq C K \quad ; \quad\|A\|_{\ell^{\infty}\left(\left\{n_{*}(K)-1, n_{*}(K), \ldots\right\}\right)} \leq C K .
$$


We denote by $\Omega^{\prime}$ the complex disk centered at the origin and of radius $\frac{1}{3 C K}$. Thus, if $\rho \in \Omega^{\prime}$, the operator $\operatorname{Id}-\rho A$ is invertible and $w=(\operatorname{Id}-\rho A)^{-1} \rho W^{0}$. It follows from (5.7) that

$$
\|w\|_{\ell^{\infty}\left(\left\{n_{*}(K)-1, n_{*}(K), \ldots\right\}\right)} \leq \frac{|\rho| C K}{1-|\rho| C K} .
$$

Therefore, $w$ is bounded in $\ell^{\infty}\left(\left\{n_{*}(K)-1, n_{*}(K), \ldots\right\}\right)$ by $\frac{1}{2}$ and $1+w_{n}>0$ for all $n \geq n_{*}(K)-1$. It also follows that $w$ is analytic in $\Omega^{\prime}$. Its derivative is

$$
\frac{\mathrm{d} w}{\mathrm{~d} \rho}(\rho)=(\operatorname{Id}-\rho A)^{-1} W^{0}+(\operatorname{Id}-\rho A)^{-2} \rho A W^{0} .
$$

Using (5.7), we get for every $\rho \in \Omega^{\prime}$

$$
\left\|\frac{\mathrm{d} w}{\mathrm{~d} \rho}(\rho)-W^{0}\right\|_{\ell^{\infty}\left(\left\{n_{*}(K)-1, n_{*}(K), \ldots\right\}\right)} \leq 4(C K)^{2}|\rho| .
$$

The proof of the proposition is complete.

\subsection{Matching.}

We consider $I=[-1 /(3 \widetilde{C} K \log K), 1 /(3 \widetilde{C} K \log K)]$ and $K$ large enough so that $\widetilde{C} \log K>C$. With this choice for the interval $I$, Propositions 5.1 and 5.2 apply for any $\rho \in I$. We will match the solutions obtained in the two previous subsections in the set $\left\{n_{*}(K)-1, n_{*}(K)\right\}$, namely $u_{n}^{0}\left(1+\delta_{n}(\rho)\right)$ for $n \leq n_{*}(K)$ and $1+w_{n}(\rho)$ for $n \geq n_{*}(K)-1$. We will prove that there is a unique $\rho \in I$ such that there exists a nonzero constant $b$ such that for $n=n_{*}(K)-1$ and $n=n_{*}(K)$,

$$
u_{n}^{0}\left(1+\delta_{n}(\rho)\right)=b\left(1+w_{n}(\rho)\right) .
$$

We have the following proposition.

\section{Proposition 5.3.}

Define the function $f$ by

$$
\begin{aligned}
f(\rho)= & u_{n_{*}(K)-1}^{0}\left(1+\delta_{n_{*}(K)-1}(\rho)\right)\left(1+w_{n_{*}(K)}(\rho)\right) \\
& -u_{n_{*}(K)}^{0}\left(1+\delta_{n_{*}(K)}(\rho)\right)\left(1+w_{n_{*}(K)-1}(\rho)\right) .
\end{aligned}
$$

The minimal positive zero $\tilde{\rho}_{0}$ of $f$ satisfies

$$
\begin{aligned}
\tilde{\rho}_{0}= & \frac{\left(1-\frac{\mu_{1}}{\lambda_{1}}\right) \sqrt{\frac{\mu_{1}}{\lambda_{1}}} \sqrt{K H^{\prime \prime}\left(x_{*}\right)} x_{*} \tilde{\lambda}\left(x_{*}\right)}{\sqrt{2 \pi}} e^{K \int_{\frac{1}{K}}^{\frac{n_{*}(K)}{K}} \log \frac{\tilde{\mu}(x)}{\lambda(x)} \mathrm{d} x} \\
& \times\left(1+\mathcal{O}\left(\frac{(\log K)^{3}}{\sqrt{K}}\right)\right)
\end{aligned}
$$

where $H$ is defined in (2.8). 
Proof. We are going to find a symmetric interval centered around 0 that contains a unique solution of $f(\rho)=0$. Define the auxiliary function $g(\rho)=$ $f(\rho)-f(0)$. One can check, using Propositions 5.1 and 5.2 and Lemma 9.6 that for all $\rho \in I$ one has

$$
\left|\frac{\mathrm{d} g}{\mathrm{~d} \rho}(\rho)-D(K)\right| \leq 39\left(\left|u_{n_{*}(K)}^{0}-u_{n_{*}(K)-1}^{0}\right| \widetilde{C} K \log K+C \widetilde{C}^{2}|\rho| K^{2}(\log K)^{2}\right)
$$

where

$$
D(K)=u_{n_{*}(K)}^{0}\left(W_{n_{*}(K)}^{0}+\Delta_{n_{*}(K)-1}^{0}-\Delta_{n_{*}(K)}^{0}\right)>0 .
$$

Let

$$
\eta(K)=\frac{10}{3} \frac{u_{n_{*}(K)}^{0}-u_{n_{*}(K)-1}^{0}}{D(K)}>0 .
$$

For all $K$ large enough we have, using Lemma 9.6 items 1 and 4,

$$
\eta(K) \leq \mathcal{O}(\sqrt{K}) e^{-c K} .
$$

Hence

$$
\begin{aligned}
& \inf _{|\rho|<\eta(K)} \frac{\mathrm{d} g}{\mathrm{~d} \rho}(\rho) \\
& \geq D(K)-39 \widetilde{C}\left(K \log K\left|u_{n_{*}(K)}^{0}-u_{n_{*}(K)-1}^{0}\right|+C \widetilde{C} \eta(K) K^{2}(\log K)^{2}\right) \\
& \geq \frac{D(K)}{3}
\end{aligned}
$$

for all $K$ large enough by Lemma 9.6. Therefore the function $g$ is monotone increasing in the interval $(-\eta(K), \eta(K))$ and, since $g(0)=0$, we have

$$
\begin{aligned}
& {\left[-\frac{10}{9}\left(u_{n_{*}(K)}^{0}-u_{n_{*}(K)-1}^{0}\right), \frac{10}{9}\left(u_{n_{*}(K)}^{0}-u_{n_{*}(K)-1}^{0}\right)\right]} \\
& =\left[-\frac{\eta(K) D(K)}{3}, \frac{\eta(K) D(K)}{3}\right] \subset g([-\eta(K), \eta(K)]) .
\end{aligned}
$$

Now because

$$
-f(0)=u_{n_{*}(K)-1}^{0}-u_{n_{*}(K)}^{0} \in\left[-\frac{10}{9}\left(u_{n_{*}(K)}^{0}-u_{n_{*}(K)-1}^{0}\right), \frac{10}{9}\left(u_{n_{*}(K)}^{0}-u_{n_{*}(K)-1}^{0}\right)\right]
$$

we have

$$
-f(0) \in g([-\eta(K), \eta(K)]) .
$$

This implies that the equation $g(\rho)=-f(0)$ has a unique solution $\tilde{\rho}_{0}$ in $[-\eta(K), \eta(K)]$. (This is a special instance of a more general result on quantitative estimates in the inverse function theorem derived in [22].) 
It follows from (5.8) that for all $\rho \in[-\eta(K), \eta(K)]$

$$
\begin{aligned}
& |f(\rho)-f(0)-D(K) \rho| \\
& \leq 39\left(\left|u_{n_{*}(K)}^{0}-u_{n_{*}(K)-1}^{0}\right| \widetilde{C}|\rho| K \log K+\frac{1}{2} C \widetilde{C}^{2}|\rho|^{2} K^{2} \log ^{2} K\right)
\end{aligned}
$$

which implies that

$$
\begin{aligned}
& \left|\frac{u_{n_{*}(K)}^{0}-u_{n_{*}(K)-1}^{0}}{D(K)}-\tilde{\rho}_{0}\right| \\
& \leq \frac{39}{D(K)}\left(\left|u_{n_{*}(K)}^{0}-u_{n_{*}(K)-1}^{0}\right| \widetilde{C} \eta(K) K \log K+\frac{1}{2} C \widetilde{C}^{2} \eta(K)^{2} K^{2} \log ^{2} K\right) .
\end{aligned}
$$

Using (5.10) and statements 1 and 4 in Lemma 9.6, the proposition follows.

We now end the proof of Theorem 3.2 . We define a sequence $\tilde{\varphi}$ by

$$
\begin{cases}\tilde{\varphi}_{n}=u_{n}^{0}\left(1+\delta_{n}\left(\tilde{\rho}_{0}\right)\right) & \text { for } n \leq n_{*}(K) \\ \tilde{\varphi}_{n}=b\left(1+w_{n}\left(\tilde{\rho}_{0}\right)\right) & \text { for } n \geq n_{*}(K)\end{cases}
$$

where $\delta_{n}\left(\tilde{\rho}_{0}\right)$ and $w_{n}\left(\tilde{\rho}_{0}\right)$ are defined in Propositions 5.1 and 5.2, and

$$
b=\frac{u_{n_{*}(K)}^{0}\left(1+\delta_{n_{*}(K)}\left(\tilde{\rho}_{0}\right)\right)}{1+w_{n_{*}(K)}\left(\tilde{\rho}_{0}\right)} .
$$

It also follows from these propositions that $\tilde{\varphi}$ is bounded and hence belongs to $\ell^{2}(\pi)$. In addition, we get for $n \geq 1$

$$
\lambda_{n} \tilde{\varphi}_{n+1}+\mu_{n} \tilde{\varphi}_{n-1} \mathbb{1}_{\{n \geq 2\}}-\left(\lambda_{n}+\mu_{n}\right) \tilde{\varphi}_{n}=-\tilde{\rho}_{0} \tilde{\varphi}_{n} .
$$

Let us consider the sequence $\left(\tilde{\varphi}^{(k)}\right)_{k \geq 1}$ of elements in $\ell^{2}(\pi)$ defined by $\tilde{\varphi}_{n}^{(k)}=$ $\tilde{\varphi}_{n} \mathbb{1}_{\{n \leq k\}}$. Remark that for all $k \geq 1, \tilde{\varphi}^{(k)} \in \mathscr{D}$. A straightforward computation leads to

$$
\left(L \tilde{\varphi}^{(k)}\right)_{n}+\tilde{\rho}_{0} \tilde{\varphi}_{n}^{(k)}= \begin{cases}0 & \text { for } n<k \\ -\lambda_{k} \tilde{\varphi}_{k+1} & \text { for } n=k \\ \mu_{k+1} \tilde{\varphi}_{k}+\tilde{\rho}_{0} \tilde{\varphi}_{k+1} & \text { for } n=k+1 \\ 0 & \text { for } n>k+1\end{cases}
$$

Using assumptions (2.12) and (2.15), we can easily prove that

$$
\lim _{k \rightarrow \infty}\left(\left\|\tilde{\varphi}-\tilde{\varphi}^{(k)}\right\|_{\ell^{2}(\pi)}^{2}+\left\|\left(L+\tilde{\rho}_{0}\right) \tilde{\varphi}^{(k)}\right\|_{\ell^{2}(\pi)}^{2}\right)=0 .
$$

This implies that $\tilde{\varphi} \in \mathcal{D}$ and $L \tilde{\varphi}=-\tilde{\rho}_{0} \tilde{\varphi}$. By Theorem 3.1, the eigenvector $\varphi$ is positive. Hence it cannot be orthogonal in $\ell^{2}(\pi)$ to $\tilde{\varphi}$ that is strictly 
positive by Propositions 5.1 and 5.2 Since $L$ is self-adjoint, this implies that $\rho_{0}=\tilde{\rho}_{0}$ and $\varphi=\tilde{\varphi}$.

By Assumption (2.9), it follows that $K \int_{0}^{\frac{1}{K}} \log \frac{\tilde{\mu}}{\hat{\lambda}}(x) \mathrm{d} x=\log \frac{\tilde{\mu}}{\lambda}\left(\frac{1}{K}\right)+$ $\mathcal{O}\left(\frac{1}{K}\right)=\log \frac{\mu_{1}}{\lambda_{1}}+\mathcal{O}\left(\frac{1}{K}\right)$. Therefore, using Proposition 5.3 we obtain

$$
\begin{aligned}
\rho_{0}= & \frac{\left(\sqrt{\frac{\lambda_{1}}{\mu_{1}}}-\sqrt{\frac{\mu_{1}}{\lambda_{1}}}\right) \sqrt{K H^{\prime \prime}\left(x_{*}\right)} x_{*} \tilde{\lambda}\left(x_{*}\right)}{\sqrt{2 \pi}} e^{-K \int_{0}^{x_{*}} \log \frac{\tilde{\lambda}(x)}{\tilde{\mu}(x)} \mathrm{d} x} \\
& \times\left(1+\mathcal{O}\left(\frac{(\log K)^{3}}{\sqrt{K}}\right)\right) .
\end{aligned}
$$

The estimate for $\varphi$ follows from Propositions 5.1 and 5.2 ,

\section{Proof of Theorem 3.3}

\subsection{A Poincaré inequality}

The proof is based on a Poincaré inequality for the Dirichlet form defined for $y \in \mathcal{D}$ by

$$
\mathscr{E}(y)=-\langle y, L y\rangle_{\pi} .
$$

Recall that $\varphi$ is the eigenvector associated to the maximal eigenvalue $-\rho_{0}$ of $L$ (see Theorem 3.1).

\section{Proposition 6.1.}

For every $y \in \mathcal{D}$ such that $\langle\varphi, y\rangle_{\pi}=0$, we have

$$
\mathscr{E}(y)-\rho_{0}\|y\|_{\pi}^{2} \geq g\|y\|_{\pi}^{2}
$$

where

$$
\frac{1}{g}=\inf _{\tilde{n} \geq 1}\left(\sum_{n=1}^{\tilde{n}} \frac{1}{\pi_{n} \lambda_{n} \varphi_{n} \varphi_{n+1}} \sum_{q=1}^{n} \pi_{q} \varphi_{q}^{2}+\sum_{n=\tilde{n}+1}^{\infty} \frac{1}{\pi_{n} \lambda_{n} \varphi_{n} \varphi_{n+1}} \sum_{q=n+1}^{\infty} \pi_{q} \varphi_{q}^{2}\right) .
$$

Proof. Take any $y \in \mathscr{D}$. This implies that there exists some integer $N$ such that $y_{n}=0$ for all $n>N$. We then have

$$
\begin{aligned}
\|y\|_{\pi}^{2} & =\sum_{n=1}^{N} \bar{y}_{n} \pi_{n} y_{n}=\sum_{n=1}^{N} \frac{\bar{y}_{n}}{\varphi_{n}} y_{n} \pi_{n} \varphi_{n} \\
& =\sum_{n=1}^{N} \frac{\bar{y}_{n}}{\varphi_{n}}\left(\sum_{q=1}^{n} y_{q} \pi_{q} \varphi_{q}-\sum_{q=1}^{n-1} y_{q} \pi_{q} \varphi_{q}\right)
\end{aligned}
$$


where by convention $\sum_{1}^{0}=0$. (Recall that $\bar{y}_{n}$ is the complex conjugate of $y_{n}$.) Hence, since $y_{N+1}=0$,

$$
\|y\|_{\pi}^{2}=\sum_{n=1}^{N}\left(\frac{\bar{y}_{n}}{\varphi_{n}}-\frac{\bar{y}_{n+1}}{\varphi_{n+1}}\right) \sum_{q=1}^{n} y_{q} \pi_{q} \varphi_{q} .
$$

By Cauchy-Schwarz inequality we get

$$
\begin{aligned}
\|y\|_{\pi}^{2} & =\sum_{n=1}^{N} \sqrt{\pi_{n} \lambda_{n} \varphi_{n} \varphi_{n+1}}\left(\frac{\bar{y}_{n}}{\varphi_{n}}-\frac{\bar{y}_{n+1}}{\varphi_{n+1}}\right)\left(\frac{1}{\sqrt{\pi_{n} \lambda_{n} \varphi_{n} \varphi_{n+1}}} \sum_{q=1}^{n} y_{q} \pi_{q} \varphi_{q}\right) \\
& \leq \sqrt{T_{1}} \sqrt{T_{2}}
\end{aligned}
$$

where

$$
T_{1}:=\sum_{n=1}^{N} \pi_{n} \lambda_{n} \varphi_{n} \varphi_{n+1}\left|\frac{y_{n}}{\varphi_{n}}-\frac{y_{n+1}}{\varphi_{n+1}}\right|^{2}
$$

and

$$
T_{2}:=\sum_{n=1}^{N} \frac{1}{\pi_{n} \lambda_{n} \varphi_{n} \varphi_{n+1}}\left|\sum_{q=1}^{n} y_{q} \pi_{q} \varphi_{q}\right|^{2} .
$$


Using that $y_{N+1}=0$ and (2.11) we obtain

$$
\begin{aligned}
T_{1}= & \sum_{n=1}^{N} \lambda_{n} \pi_{n} \frac{\varphi_{n+1}}{\varphi_{n}}\left|y_{n}\right|^{2}+\sum_{n=1}^{N} \lambda_{n} \pi_{n} \frac{\varphi_{n}}{\varphi_{n+1}}\left|y_{n+1}\right|^{2}-\sum_{n=1}^{N} \lambda_{n} \pi_{n} \bar{y}_{n} y_{n+1} \\
& -\sum_{n=1}^{N} \lambda_{n} \pi_{n} \bar{y}_{n+1} y_{n} \\
= & \sum_{n=1}^{N} \lambda_{n} \pi_{n} \frac{\varphi_{n+1}}{\varphi_{n}}\left|y_{n}\right|^{2}+\sum_{n=2}^{N+1} \lambda_{n-1} \pi_{n-1} \frac{\varphi_{n-1}}{\varphi_{n}}\left|y_{n}\right|^{2}-\sum_{n=1}^{N} \lambda_{n} \pi_{n} \bar{y}_{n} y_{n+1} \\
& -\sum_{n=1}^{N} \lambda_{n} \pi_{n} \bar{y}_{n+1} y_{n} \\
= & \sum_{n=2}^{N}\left(\lambda_{n} \pi_{n} \frac{\varphi_{n+1}}{\varphi_{n}}+\lambda_{n-1} \pi_{n-1} \frac{\varphi_{n-1}}{\varphi_{n}}\right)\left|y_{n}\right|^{2}+\lambda_{1} \pi_{1} \frac{\varphi_{2}}{\varphi_{1}}\left|y_{1}\right|^{2} \\
& -\sum_{n=1}^{N} \lambda_{n} \pi_{n} \bar{y}_{n} y_{n+1}-\sum_{n=1}^{N} \lambda_{n} \pi_{n} \bar{y}_{n+1} y_{n} \\
= & \sum_{n=2}^{N}\left(\lambda_{n} \pi_{n} \frac{\varphi_{n+1}}{\varphi_{n}}+\mu_{n} \pi_{n} \frac{\varphi_{n-1}}{\varphi_{n}}\right)\left|y_{n}\right|^{2}+\lambda_{1} \pi_{1} \frac{\varphi_{2}}{\varphi_{1}}\left|y_{1}\right|^{2} \\
& -\sum_{n=1}^{N} \lambda_{n} \pi_{n} \bar{y}_{n} y_{n+1}-\sum_{n=1}^{N} \lambda_{n} \pi_{n} \bar{y}_{n+1} y_{n} \\
= & \sum_{n=1}^{N}\left(\lambda_{n}+\mu_{n}\right) \pi_{n}\left|y_{n}\right|^{2}-\sum_{n=1}^{N} \lambda_{n} \pi_{n} \bar{y}_{n} y_{n+1}-\sum_{n=1}^{N} \lambda_{n} \pi_{n} \bar{y}_{n+1} y_{n} \\
& \sum_{n=1}^{N} \pi_{n}\left|y_{n}\right|^{2}
\end{aligned}
$$

since for $n \geq 2$

$$
\lambda_{n} \varphi_{n+1}+\mu_{n} \varphi_{n-1}-\left(\lambda_{n}+\mu_{n}\right) \varphi_{n}=-\rho_{0} \varphi_{n}
$$

and

$$
\lambda_{1} \varphi_{2}-\left(\lambda_{1}+\mu_{1}\right) \varphi_{1}=-\rho_{0} \varphi_{1} .
$$

Note also that (since $y_{N+1}=0$ and using (2.11))

$$
\begin{gathered}
\sum_{n=1}^{N} \lambda_{n} \pi_{n} \bar{y}_{n+1} y_{n}=\sum_{p=2}^{N+1} \lambda_{p-1} \pi_{p-1} \bar{y}_{p} y_{p-1} \\
=\sum_{p=2}^{N+1} \mu_{p} \pi_{p} \bar{y}_{p} y_{p-1}=\sum_{p=2}^{N} \mu_{p} \pi_{p} \bar{y}_{p} y_{p-1} .
\end{gathered}
$$


Therefore

$$
T_{1} \leq \mathscr{E}(y)-\rho_{0}\|y\|_{\pi}^{2},
$$

and we get from (6.3) and the previous estimate

$$
\|y\|_{\pi}^{2} \leq \sqrt{\mathscr{E}(y)-\rho_{0}\|y\|_{\pi}^{2}} \sqrt{T_{2}} .
$$

We now derive an upper bound for $T_{2}$. We now use the assumption that $y$ is such that $\langle\varphi, y\rangle_{\pi}=0$ on the top of being such that $y_{n}=0$ for all $n \geq N+1$. In other words

$$
\sum_{q=1}^{N} y_{q} \pi_{q} \varphi_{q}=\sum_{q=1}^{\infty} y_{q} \pi_{q} \varphi_{q}=\langle\varphi, y\rangle_{\pi}=0 .
$$

Let $\tilde{n}$ be a fixed integer over which we will optimize later on. Then we get, using Cauchy-Schwarz inequality,

$$
\begin{aligned}
T_{2} \leq & \sum_{n=1}^{\infty} \frac{1}{\pi_{n} \lambda_{n} \varphi_{n} \varphi_{n+1}}\left|\sum_{q=1}^{n} y_{q} \varphi_{q} \pi_{q}\right|^{2} \\
= & \sum_{n=1}^{n} \frac{1}{\pi_{n} \lambda_{n} \varphi_{n} \varphi_{n+1}}\left|\sum_{q=1}^{n} y_{q} \varphi_{q} \pi_{q}\right|^{2}+\sum_{n=\tilde{n}+1}^{\infty} \frac{1}{\pi_{n} \lambda_{n} \varphi_{n} \varphi_{n+1}}\left|\sum_{q=1}^{n} y_{q} \varphi_{q} \pi_{q}\right|^{2} \\
= & \sum_{n=1}^{n} \frac{1}{\pi_{n} \lambda_{n} \varphi_{n} \varphi_{n+1}}\left|\sum_{q=1}^{n} y_{q} \varphi_{q} \pi_{q}\right|^{2}+\sum_{n=\tilde{n}+1}^{\infty} \frac{1}{\pi_{n} \lambda_{n} \varphi_{n} \varphi_{n+1}}\left|\sum_{q=n+1}^{\infty} y_{q} \varphi_{q} \pi_{q}\right|^{2} \\
\leq & \sum_{n=1}^{n} \frac{1}{\pi_{n} \lambda_{n} \varphi_{n} \varphi_{n+1}}\left(\sum_{q=1}^{n}\left|y_{q}\right|^{2} \pi_{q}\right)\left(\sum_{q=1}^{n} \varphi_{q}^{2} \pi_{q}\right) \\
& +\sum_{n=\tilde{n}+1}^{\infty} \frac{1}{\pi_{n} \lambda_{n} \varphi_{n} \varphi_{n+1}}\left(\sum_{q=n+1}^{\infty}\left|y_{q}\right|^{2} \pi_{q}\right)\left(\sum_{q=n+1}^{\infty} \varphi_{q}^{2} \pi_{q}\right) \\
\leq & \left(\sum_{n=1}^{\tilde{n}} \frac{1}{\pi_{n} \lambda_{n} \varphi_{n} \varphi_{n+1}} \sum_{q=1}^{n} \varphi_{q}^{2} \pi_{q}+\sum_{n=\tilde{n}+1}^{\infty} \frac{1}{\pi_{n} \lambda_{n} \varphi_{n} \varphi_{n+1}} \sum_{q=n+1}^{\infty} \varphi_{q}^{2} \pi_{q}\right)\|y\|_{\pi}^{2} .
\end{aligned}
$$

We used (6.5) for the second equality, that is, $\sum_{q=1}^{n} y_{q} \varphi_{q} \pi_{q}=-\sum_{q=n+1}^{\infty} y_{q} \varphi_{q} \pi_{q}$. Combining (6.4) and the previous bound we thus get that, if $\langle\varphi, y\rangle_{\pi}=0$,

$$
\|y\|_{\pi}^{2} \leq \sqrt{\mathscr{E}(y)-\rho_{0}\|y\|_{\pi}^{2}} \frac{1}{\sqrt{g}}\|y\|_{\pi}
$$

where $g$ has been defined in (6.2). This implies (6.1) on $\mathcal{D}$ by closure. 


\subsection{Lower estimate for the spectral gap}

\section{LEMMA 6.2.}

The spectral gap is bounded below by $g$ defined in (6.2):

$$
\rho_{1}-\rho_{0} \geq g
$$

Proof. Let us consider an eigenvector $y \in \mathcal{D}$ with eigenvalue $-\rho_{1}$. Since $L$ is self-adjoint in $\ell^{2}(\pi)$, we have $\langle\varphi, y\rangle=0$. Therefore we get from inequality (6.1) in Proposition 6.1

$$
-\rho_{1}\|y\|_{\pi}^{2}=\langle y, L y\rangle_{\pi} \leq-\left(\rho_{0}+g\right)\|y\|_{\pi}^{2}
$$

and the result follows.

From what precedes, the proof of Theorem 3.3 boils down to prove the following proposition.

\section{Proposition 6.3.}

For all $K \geq 2, g \geq \frac{\mathcal{O}(1)}{\log K}$ where $g$ is defined in (6.2).

Before giving the proof of this proposition, we introduce the following technical quantities. Let

$$
x_{* *}=\inf \left\{x \in \mathbb{R}+: \frac{\tilde{\lambda}(x)}{\tilde{\mu}(x)}<\frac{1}{2}\right\} .
$$

Observe that $x_{* *}<\infty$ because of $(2.2)$. Also observe that $x_{*}<x_{* *}$ by the assumptions made on the functions $\tilde{\lambda}$ and $\tilde{\mu}$. We also define

$$
n_{* *}(K)=\left\lfloor x_{* *} K\right\rfloor .
$$

We will also need to introduce an integer $n_{* * *}(K)$ that is defined as follows. By the assumptions made on the functions $\tilde{\mu}$ and $\tilde{\lambda}$ (see (2.3) and (2.4)), there exists a number $\theta$ such that

$$
\frac{\tilde{\mu}(0)}{\tilde{\lambda}(0)}<\theta<1
$$

Thus we can define the following real number (that is strictly smaller than $\left.x_{*}\right)$.

$$
x_{* * *}=\sup \left\{x: \frac{\tilde{\mu}(x)}{\tilde{\lambda}(x)} \leq \theta\right\} .
$$

Then we define the integer

$$
n_{* * *}(K)=\left\lfloor x_{* * *} K\right\rfloor .
$$


By definition

$$
n_{* * *}(K) \leq n_{*}(K) \leq n_{* *}(K) .
$$

We now turn to the proof of Proposition 6.3.

Proof. From Lemma 9.3 and Theorem 3.2 , we have

$$
\sup _{n \in \mathbb{N}^{*}, K}\left|\varphi_{n}\right|<+\infty \quad \text { and } \sup _{n \in \mathbb{N}^{*}, K}\left|\varphi_{n}^{-1}\right|<+\infty .
$$

Therefore

$$
\frac{1}{g} \leq \mathcal{O}(1)\left(\sum_{n=1}^{n_{*}(K)} \frac{1}{\pi_{n} \lambda_{n}} \sum_{q=1}^{n} \pi_{q}+\sum_{n=n_{*}(K)+1}^{\infty} \frac{1}{\pi_{n} \lambda_{n}} \sum_{q=n+1}^{\infty} \pi_{q}\right) .
$$

We now derive an upper bound for each sum.

We first deal with the second sum in (6.10). To this end we write

$$
\sum_{n=n_{*}(K)+1}^{\infty} \frac{1}{\pi_{n} \lambda_{n}} \sum_{q=n+1}^{\infty} \pi_{q}=S_{1}+S_{2}+S_{3}
$$

where

$$
\begin{aligned}
& S_{1}=\sum_{n=n_{* *}(K)}^{\infty} \sum_{q=n+1}^{\infty} \frac{\pi_{q}}{\lambda_{n} \pi_{n}}, \quad S_{2}=\sum_{n=n_{*}(K)+1}^{n_{* *(K)}} \sum_{q=n_{* *}(K)+1}^{\infty} \frac{\pi_{q}}{\lambda_{n} \pi_{n}} \\
& \text { and } S_{3}=\sum_{n=n_{*}(K)+1}^{n_{* *}(K)} \sum_{q=n+1}^{n_{* *}(K)} \frac{\pi_{q}}{\lambda_{n} \pi_{n}}
\end{aligned}
$$

where $n_{* *}(K)$ is defined in (6.7). Using Young's inequality and Lemma 9.1 we first get

$$
S_{1} \leq \sum_{n=n_{* *}(K)}^{\infty} \sum_{q=n+1}^{\infty} \frac{1}{\mu_{q}}\left(\frac{1}{2}\right)^{q-n-1} \leq \mathcal{O}(1) \sum_{q=n_{* *}(K)}^{\infty} \frac{1}{\mu_{q}} \leq \mathcal{O}(1) .
$$

Next we have

$$
\begin{aligned}
S_{2} & =\sum_{n=n_{*}(K)+1}^{n_{* *(K)}} \sum_{q=n_{* *}(K)+1}^{\infty} \frac{\pi_{q}}{\lambda_{n} \pi_{n}} \leq \sum_{n=n_{*}(K)+1}^{n_{* *(K)}} \sum_{q=n_{* *(K)+1}}^{\infty} \frac{1}{\mu_{q}}\left(\frac{1}{2}\right)^{q-n_{* *}(K)-1} \\
& \leq \frac{\mathcal{O}(1)}{K} \sum_{n=n_{*}(K)+1}^{n_{* *}(K)} \sum_{q=n_{* *}(K)+1}^{\infty}\left(\frac{1}{2}\right)^{q-n_{* *}(K)-1} \leq \mathcal{O}(1) .
\end{aligned}
$$

We used several facts: $\left(\mu_{q}\right)$ is increasing, $\tilde{\mu}(x) \geq \tilde{\mu}(0)>0$, and the integers $n_{*}(K), n_{* *}(K)$ are of order $K$. Finally we have, using Lemma 9.4 and the 
numbers $\Lambda_{n, m}$ defined just before that lemma,

$$
\begin{aligned}
S_{3} & =\sum_{n=n_{*}(K)+1}^{n_{* *}(K)} \sum_{q=n+1}^{n_{* *(}(K)} \frac{\pi_{q}}{\lambda_{n} \pi_{n}}=\sum_{n=n_{*}(K)+1}^{n_{* *}(K)} \sum_{q=n+1}^{n_{* *}(K)} \frac{\Lambda_{q, n+1}}{\mu_{q}} \\
& \leq \frac{\mathcal{O}(1)}{K} \sum_{n=n_{*}(K)}^{n_{* *}(K)} \sum_{q=n+1}^{n_{* *}(K)} e^{-K\left(H\left(\frac{q}{K}\right)-H\left(\frac{n+1}{K}\right)\right)} .
\end{aligned}
$$

For $x_{*} \leq s \leq x_{* *}$ (see (6.6) for the definition of $x_{* *}$ ) we have for some positive constant $\hat{c}$

$$
\log \frac{\tilde{\mu}(s)}{\tilde{\lambda}(s)} \leq \hat{c}\left(s-x_{*}\right) .
$$

Hence we get

$$
\begin{aligned}
S_{3} & \leq \frac{\mathcal{O}(1)}{K} \sum_{n=n_{*}(K)+1}^{n_{* *(K)}} \sum_{q=n+1}^{n_{* *(K)}} e^{-\frac{\hat{c}}{2 K}\left(\left(q-K x_{*}\right)^{2}-\left(n+1-K x_{*}\right)^{2}\right)} \\
& \leq \mathcal{O}(1)+\frac{\mathcal{O}(1)}{K} \sum_{n=n_{*}(K)+1}^{n_{* *}(K)} \sum_{q=n+2}^{n_{* *}(K)} e^{-\frac{\hat{c}}{2 K}(q-n-1)\left(q+n+1-2 K x_{*}\right)}
\end{aligned}
$$

where we have isolated the term $q=n+1$ that gives $\mathcal{O}(1)$. We introduce the new variables $p=q+n+1$ and $r=q-n-1$ to get

$$
\begin{aligned}
S_{3} & \leq \mathcal{O}(1)+\frac{\mathcal{O}(1)}{K} \sum_{r=1}^{n_{* *}(K)-n_{*}(K)-2} \sum_{p=2 n_{*}(K)+r+2}^{2 n_{* *}(K)-r} e^{-\frac{\hat{c} r\left(p-2 K x_{*}\right)}{2 K}} \\
& =\mathcal{O}(1)+\frac{\mathcal{O}(1)}{K} \sum_{r=1}^{n_{* *(K)-n_{*}(K)}} \frac{1}{1-e^{-\frac{\hat{c} r}{2 K}}} \\
& \leq \mathcal{O}(1)+\frac{\mathcal{O}(1)}{K} \sum_{r=1}^{n_{* *}^{(K)-n_{*}(K)}}\left(\frac{K}{r}+1\right) \\
& \leq \mathcal{O}(1)+\mathcal{O}(1) \sum_{r=1}^{n_{* *}(K)-n_{*}(K)} \frac{1}{r} \leq \mathcal{O}(1) \log K .
\end{aligned}
$$

We now turn to the sum running from 1 to $n_{*}(K)$ in (6.10). We write

$$
\sum_{n=1}^{n_{*}(K)} \frac{1}{\pi_{n} \lambda_{n}} \sum_{q=1}^{n} \pi_{q}=\sum_{n=1}^{n_{*}(K)} \sum_{q=1}^{n} \frac{\Lambda_{n+1, q}}{\mu_{q}}=\hat{S}_{1}+\hat{S}_{2}+\hat{S}_{3}
$$

where

$$
\begin{aligned}
& \hat{S}_{1}=\sum_{n=1}^{n_{* * *(K)}} \sum_{q=1}^{n} \frac{\pi_{q}}{\lambda_{n} \pi_{n}}, \quad \hat{S}_{2}=\sum_{n=n_{* * *}(K)}^{n_{*(K)}} \sum_{q=1}^{n_{* * *(K)}} \frac{\Lambda_{n+1, q}}{\mu_{q}} \\
& \text { and } \hat{S}_{3}=\sum_{n=n_{* * *}(K)}^{n_{*}(K)} \sum_{q=n_{* * *}(K)}^{n} \frac{\Lambda_{n+1, q}}{\mu_{q}} .
\end{aligned}
$$


By using (2.3) and inverting the order of summations we get

$$
\hat{S}_{1} \leq \sum_{n=1}^{n_{* * *}(K)} \sum_{q=1}^{n} \frac{1}{\mu_{q}} \theta^{n-q} \leq \sum_{n=1}^{n_{* * *(K)}} \sum_{q=1}^{n} \frac{1}{\tilde{\mu}(0) q} \theta^{n-q} \leq \mathcal{O}(1) \log K
$$

where $n_{* * *}(K)$ is defined in (6.9). We estimate $\hat{S}_{2}$ as follows.

$$
\hat{S}_{2} \leq \mathcal{O}(1) \sum_{n=n_{* * *}(K)}^{n_{*}(K)} \sum_{q=1}^{n_{* * *}(K)} \frac{1}{q} \theta^{\left(n_{* * *}(K)-q\right)} \leq \mathcal{O}(1) .
$$

The last estimate follows by splitting the second sum from 1 to $n_{* * *(K)} / 2$ and from $n_{* * *(K)} / 2$ to $n_{* * *(K)}-1$.

Finally, we have the estimates

$$
\hat{S}_{3} \leq \frac{\mathcal{O}(1)}{K} \sum_{n=n_{* * *}(K)}^{n_{*}(K)} \sum_{q=n_{* * *}(K)}^{n} e^{K\left(H\left(\frac{n}{K}\right)-H\left(\frac{q}{K}\right)\right)} .
$$

For $x_{* * *} \leq s \leq x_{*}$ we have

$$
\log \frac{\tilde{\mu}(s)}{\tilde{\lambda}(s)} \leq-c_{2}\left(x_{*}-s\right)
$$

for some constant $c_{2}>0$, hence

$$
\begin{aligned}
\hat{S}_{3} & \leq \frac{\mathcal{O}(1)}{K} \sum_{n=n_{* * *}(K)}^{n_{*}(K)} \sum_{q=n_{* * *}(K)}^{n} e^{-\frac{c_{2}}{2 K}\left(\left(q-K x_{*}\right)^{2}-\left(n-K x_{*}\right)^{2}\right)} \\
& \leq \frac{\mathcal{O}(1)}{K} \sum_{n=n_{* * *}(K)}^{n_{*}(K)} \sum_{q=n_{* * *}(K)}^{n} e^{-\frac{c_{2}}{2 K}(n-q)\left(2 K x_{*}-q-n\right)} .
\end{aligned}
$$

We now use the variables $p=q+n$ and $r=q-n$,

$$
\begin{aligned}
\hat{S}_{3} & \leq \mathcal{O}(1)+\frac{\mathcal{O}(1)}{K} \sum_{r=1}^{n_{*}(K)-n_{* * *}(K)} \sum_{p=2 n_{* * *}(K)+r}^{2 n_{*}(K)-r} e^{-c_{2} r \frac{\left(p-2 K x_{*}\right)}{2 K}} \\
& =\mathcal{O}(1)+\frac{\mathcal{O}(1)}{K} \sum_{r=1}^{n_{*}(K)-n_{* * *(K)}} \frac{1}{1-e^{-\frac{c_{2} r}{2 K}}} \\
& \leq \mathcal{O}(1)+\frac{\mathcal{O}(1)}{K} \sum_{r=1}^{n_{*}(K)-n_{* * *}(K)}\left(\frac{K}{r}+1\right) \\
& \leq \mathcal{O}(1)+\mathcal{O}(1) \sum_{r=1}^{n_{*}(K)-n_{* * *(K)}} \frac{1}{r} \leq \mathcal{O}(1) \log K .
\end{aligned}
$$

Gathering all the bounds, we get the desired result. 


\section{Proof of Theorem 3.6}

\subsection{Preliminary estimates}

We first derive some useful estimates. Recall that the constant $c$ has been defined in (3.4).

\section{Proposition 7.1.}

For all $K>1$ we have

$$
\left|\frac{\langle\varphi, \mathbb{1}\rangle_{\pi}}{\|\varphi\|_{\pi}^{2}}-\frac{1}{u_{n_{*}(K)}^{0}}\right| \leq \mathcal{O}(1) K^{\frac{3}{2}} \log K e^{-c K} .
$$

Proof. Recall that

$$
V_{n}= \begin{cases}u_{n}^{0} & \text { if } n \leq n_{*}(K) \\ u_{n_{*}(K)}^{0} & \text { if } n \geq n_{*}(K) .\end{cases}
$$

Assume that $K$ is large enough so that Propositions 5.1, 5.2 and Lemma 9.3 apply. We obtain

$$
1-\mathcal{O}(1) \rho_{0}(K) K \log K \leq \frac{\langle\varphi, \mathbb{1}\rangle_{\pi}}{\|\varphi\|_{\pi}^{2}} \frac{\|V\|_{\pi}^{2}}{\langle V, \mathbb{1}\rangle_{\pi}} \leq 1+\mathcal{O}(1) \rho_{0}(K) K \log K .
$$

Observe that $\|\mathbb{1}\|_{\pi}^{2}=\sum_{j=1}^{\infty} \pi_{j}$ and

$$
\langle V, \mathbb{1}\rangle_{\pi}-u_{n_{*}(K)}^{0}\|\mathbb{1}\|_{\pi}^{2}=\sum_{j=1}^{n_{*}(K)-1}\left(u_{j}^{0}-u_{n_{*}(K)}^{0}\right) \pi_{j} .
$$

Now using (3.3) we get for all $j \leq n_{*}(K)-1$

$$
u_{n_{*}(K)}^{0}-u_{j}^{0}=\sum_{\ell=j}^{n_{*}(K)-1} \frac{1}{\lambda_{\ell} \pi_{\ell}}
$$

Hence

$$
\langle V, \mathbb{1}\rangle_{\pi}-u_{n_{*}(K)}^{0}\|\mathbb{1}\|_{\pi}^{2}=\sum_{j=1}^{n_{*}(K)-1} \sum_{\ell=j}^{n_{*}(K)-1} \frac{\Lambda_{\ell+1, j}}{\mu_{j}} .
$$

We split this sum into three sums, $s_{1}, s_{2}$ and $s_{3}$, that we define and estimate as follows. We have

$$
s_{1}=\sum_{j=1}^{n_{* * *(K)}-1} \sum_{\ell=j}^{n_{* * *(K)}-1} \frac{\Lambda_{\ell+1, j}}{\mu_{j}} \leq \mathcal{O}(1) \log K
$$


since in this range $\Lambda_{\ell+1, j} \leq \theta^{\ell-j+1}$ and $\mu_{j} \geq j \tilde{\mu}\left(x_{*}\right)$. Next we have

$$
\begin{aligned}
s_{2} & =\sum_{j=1}^{n_{* * *(K)}-1} \sum_{\ell=n_{* * *}(K)}^{n_{*(K)}-1} \frac{\Lambda_{\ell+1, j}}{\mu_{j}} \\
& \leq \mathcal{O}(1) \sum_{j=1}^{n_{* * *(K)}-1} \frac{1}{j} \sum_{\ell=n_{* * *}(K)}^{n_{*}(K)-1} \Lambda_{\ell+1, n_{* * *}(K)+1} \Lambda_{n_{* * *}(K), j} .
\end{aligned}
$$

We use the fact that $\Lambda_{\ell+1, n_{* * *}(K)+1} \leq 1$ and $\Lambda_{n_{* * *}(K), j} \leq \theta^{n_{* * *}(K)-j}$ to get

$$
\begin{aligned}
s_{2} & \leq \mathcal{O}(1) \sum_{j=1}^{n_{* * *}(K)-1} \frac{1}{j} \sum_{\ell=n_{* * *}(K)}^{n_{*}(K)-1} \theta^{n_{* * *}(K)-j} \\
& \leq \mathcal{O}(1) K \sum_{j=1}^{n_{* * *(K)}-1} \frac{1}{j} \theta^{n_{* * *}(K)-j} \leq \mathcal{O}(1)
\end{aligned}
$$

that can be seen by estimating the sums from 1 to $n_{* * *(K)} / 2$ and from $n_{* * *(K)} / 2$ to $n_{* * *}(K)-1$. Finally

$$
\begin{aligned}
s_{3} & =\sum_{j=n_{* * *}(K)}^{n_{*}(K)-1} \sum_{\ell=j}^{n_{*}(K)-1} \frac{\Lambda_{\ell+1, j}}{\mu_{j}} \\
& \leq \mathcal{O}(1) \frac{1}{K} \sum_{j=n_{* * *}(K)}^{n_{*}(K)-1} \sum_{\ell=j}^{n_{*}(K)-1} \Lambda_{\ell+1, j} \leq \mathcal{O}(1) \log K
\end{aligned}
$$

where we first interchange the summations and then follow a very similar argument as in the estimate of $S_{3}$ in the proof of Proposition 6.3. Therefore we obtain

$$
\left|\langle V, \mathbb{1}\rangle_{\pi}-u_{n_{*}(K)}^{0}\|\mathbb{1}\|_{\pi}^{2}\right| \leq \mathcal{O}(1) \log K .
$$

Now observe that

$$
\begin{aligned}
\|V\|_{\pi}^{2}-\left(u_{n_{*}(K)}^{0}\right)^{2}\|\mathbb{1}\|_{\pi}^{2} & =\sum_{j=1}^{n_{*}(K)-1}\left(\left(u_{j}^{0}\right)^{2}-\left(u_{n_{*}(K)}^{0}\right)^{2}\right) \pi_{j} \\
& =\sum_{j=1}^{n_{*}(K)-1}\left(u_{j}^{0}-u_{n_{*}(K)}^{0}\right)\left(u_{j}^{0}+u_{n_{*}(K)}^{0}\right) \pi_{j} .
\end{aligned}
$$

Since $\left(u_{j}^{0}\right)$ is monotone increasing and using Lemma 9.3 we get

$$
\left|\|V\|_{\pi}^{2}-\left(u_{n_{*}(K)}^{0}\right)^{2}\|\mathbb{1}\|_{\pi}^{2}\right| \leq \mathcal{O}(1) \sum_{j=1}^{n_{*}(K)-1}\left(u_{n_{*}(K)}^{0}-u_{j}^{0}\right) \pi_{j} \leq \mathcal{O}(1) \log K
$$


as we have seen above.

Using (7.1) we have

$$
\begin{aligned}
& \left|\frac{\langle\varphi, \mathbb{1}\rangle_{\pi}}{\|\varphi\|_{\pi}^{2}}-\frac{1}{u_{n_{*}(K)}^{0}}\right| \leq\left|\frac{\langle\varphi, \mathbb{1}\rangle_{\pi}}{\|\varphi\|_{\pi}^{2}}-\frac{\langle V, \mathbb{1}\rangle_{\pi}}{\|V\|_{\pi}^{2}}\right|+\left|\frac{\langle V, \mathbb{1}\rangle_{\pi}}{\|V\|_{\pi}^{2}}-\frac{1}{u_{n_{*}(K)}^{0}}\right| \\
& \mathcal{O}(1) \rho_{0}(K) K \log K \frac{\langle V, \mathbb{1}\rangle_{\pi}}{\|V\|_{\pi}^{2}}+\frac{\left|\langle V, \mathbb{1}\rangle_{\pi}-u_{n_{*}(K)}^{0}\|\mathbb{1}\|_{\pi}^{2}\right|}{\|V\|_{\pi}^{2}}+\frac{\left|\|V\|_{\pi}^{2}-\left(u_{n_{*}(K)}^{0}\right)^{2}\|\mathbb{1}\|_{\pi}^{2}\right|}{u_{n_{*}(K)}^{0}\|V\|_{\pi}^{2}} .
\end{aligned}
$$

The result follows using (7.2), (17.3), Lemma 9.3, and the estimation

$$
\|V\|_{\pi}^{2} \geq \sum_{n=1}^{\infty} \pi_{n} \geq \gamma \sqrt{K} e^{c K}
$$

where the first inequality follows again from Lemma 9.3 and the definition of $V$, while the second inequality is the lower bound in statement 5 in Lemma 9.6 .

Note that for every $A \in \mathscr{P}\left(\mathbb{N}^{*}\right), \mathbb{1}_{A} \in \ell^{2}(\pi)$.

\section{Proposition 7.2.}

There exists $\bar{C}>0$ such that for all $t \geq 0$, for all $K>1$ and for all $n \in \mathbb{N}^{*}$, we have

$$
\sup _{A \in \mathscr{P}\left(\mathbb{N}^{*}\right)}\left|P_{t}(n, A)-e^{-\rho_{0} t} \varphi_{n} \frac{\langle\varphi, \mathbb{1}\rangle_{\pi}}{\|\varphi\|_{\pi}^{2}} \nu(A)\right| \leq \frac{\bar{C} K^{\frac{1}{4}} e^{\frac{c}{2} K} e^{-\rho_{1} t}}{\sqrt{\pi_{n}}}
$$

where $c$ is defined in (3.4).

Proof. Let $\mathcal{Q}$ be the spectral projection on the spectral complement of $-\rho_{0}$. By spectral theory (see e.g. [14, Theorem V.2.10, p. 260]) we have

$$
e^{t L_{1}} \mathbb{1}_{A}=e^{-\rho_{0} t} \varphi \frac{\left\langle\varphi, \mathbb{1}_{A}\right\rangle_{\pi}}{\|\varphi\|_{\pi}^{2}}+e^{t L} \mathcal{Q} \mathbb{1}_{A}
$$

Again by spectral theory and Cauchy-Schwarz inequality

$$
\left|\left\langle\mathrm{e}_{n}, e^{t L} \mathcal{Q} \mathbb{1}_{A}\right\rangle_{\pi}\right| \leq e^{-\rho_{1} t}\left\|\mathrm{e}_{n}\right\|_{\pi}\left\|\mathbb{1}_{A}\right\|_{\pi} \leq e^{-\rho_{1} t} \sqrt{\pi_{n}} \sqrt{\sum_{j=1}^{\infty} \pi_{j}} .
$$

since $\left\|\mathbb{1}_{A}\right\|_{\pi}^{2} \leq\|\mathbb{1}\|_{\pi}^{2}=\sum_{j=1}^{\infty} \pi_{j}$. The result follows from the definition of $P_{t}$ (see (3.1) ) using statement 5 of Lemma 9.6.

The estimate in Proposition 7.2 is not satisfactory for $n$ large since $\pi_{n}$ tends to 0 as $n$ tends to infinity. In fact, we can use the descent from infinity to get an estimate on the error that is uniform in $n$. 


\section{Proposition 7.3.}

There exist three strictly positive constants $a, c_{1}, C^{\prime}$ such that for all $t \geq 0$, for all $K>1$ and for all $n \in \mathbb{N}^{*}$, we have

$$
\sup _{A \in \mathscr{P}\left(\mathbb{N}^{*}\right)}\left|P_{t}(n, A)-e^{-\rho_{0} t} \varphi_{n} \frac{\langle\varphi, \mathbb{1}\rangle_{\pi}}{\|\varphi\|_{\pi}^{2}} \nu(A)\right| \leq C^{\prime}\left(K e^{-\frac{a}{4} t}+K^{\frac{3}{4}} e^{c_{1} K} e^{-\frac{\rho_{1}}{2} t}\right) .
$$

Proof. For $q \in \mathbb{N}$ define $T_{q}=\inf \left\{t \geq 0: X_{t}^{K}=q\right\}$. From the proof of Proposition 2.3 in [3] we obtain

$$
\sup _{n \geq n_{* *}(K)} \mathbb{E}_{n}\left[e^{a T_{n * *}(K)}\right] \leq \mathcal{O}(1) K
$$

where

$$
a=\inf _{K>1}\left(\sum_{j=n_{* *}(K)}^{\infty} \frac{1}{\lambda_{j} \pi_{j}} \sum_{p=j+1}^{\infty} \pi_{p}\right)^{-1} .
$$

One can prove that $a>0$ (see Lemma 9.2 for a proof).

Using Chebyshev inequality we get for all $t>0$

$$
\sup _{n \geq n_{* *}(K)} \mathbb{P}_{n}\left(T_{n_{* *}(K)} \geq \frac{t}{2}\right) \leq \mathcal{O}(1) K e^{-\frac{a}{2} t} .
$$

For every $n \geq n_{* *}(K)$, we have

$$
\begin{aligned}
P_{t}(n, A) & =\mathbb{E}_{n}\left[\mathbb{1}_{A}\left(X_{t}^{K}\right) \mathbb{1}_{\left\{T_{0}>t\right\}}\right] \\
& =\mathbb{E}_{n}\left[\mathbb{1}_{A}\left(X_{t}^{K}\right) \mathbb{1}_{\left\{T_{0}>t\right\}} \mathbb{1}_{\left\{T_{n_{*}(K)}<\frac{t}{2}\right\}}\right]+\mathcal{O}(1) K e^{-\frac{a}{2} t} .
\end{aligned}
$$

By the strong Markov property we have

$$
\begin{aligned}
& \mathbb{E}_{n}\left[\mathbb{1}_{A}\left(X_{t}^{K}\right) \mathbb{1}_{\left\{T_{0}>t\right\}} \mathbb{1}_{\left\{T_{n_{* *}(K)}<\frac{t}{2}\right\}}\right] \\
& =\mathbb{E}_{n}\left[\mathbb{E}_{n_{* *}(K)}\left(\mathbb{1}_{A}\left(X_{t-T_{n_{* *}(K)}}^{K}\right) \mathbb{1}_{\left\{T_{0}>t-T_{n_{* *}(K)}\right\}}\right) \mathbb{1}_{\left\{T_{0}>T_{n_{* *}(K)}\right\}} \mathbb{1}_{\left\{T_{n_{* *}(K)}<\frac{t}{2}\right\}}\right] .
\end{aligned}
$$

Using Proposition 7.2 and Lemma 9.4 we obtain

$$
\begin{aligned}
& \mathbb{E}_{n}\left[\mathbb{1}_{A}\left(X_{t}^{K}\right) \mathbb{1}_{\left\{T_{0}>t\right\}} \mathbb{1}_{\left\{T_{n_{* *}(K)}<\frac{t}{2}\right\}}\right] \\
& =\varphi_{n_{* *}(K)} \frac{\langle\varphi, \mathbb{1}\rangle_{\pi}}{\|\varphi\|_{\pi}^{2}} e^{-\rho_{0} t} \nu(A) \mathbb{E}_{n}\left[e^{\rho_{0} T_{n_{* *}(K)}} \mathbb{1}_{\left\{T_{n_{* *}(K)}<\frac{t}{2}\right\}}\right]+\mathcal{O}(1) K^{\frac{3}{4}} e^{c_{1} K} e^{-\frac{\rho_{1}}{2} t}
\end{aligned}
$$

where $c_{1}>0$ is a constant independent of $n, t, A$ and $K$. Using CauchySchwarz inequality we obtain, using (7.4) and (7.5),

$$
\begin{aligned}
\mathbb{E}_{n}\left[e^{\rho_{0} T_{n_{* *}(K)}} \mathbb{1}_{\left\{T_{n_{* *}(K)} \geq \frac{t}{2}\right\}}\right] & \leq\left(\mathbb{E}_{n}\left[e^{2 \rho_{0} T_{n_{* *}(K)}}\right]\right)^{\frac{1}{2}}\left(\mathbb{E}_{n}\left[\mathbb{1}_{\left\{T_{n_{* *}(K)} \geq \frac{t}{2}\right\}}\right]\right)^{\frac{1}{2}} \\
& \leq \mathcal{O}(1) K e^{-\frac{a}{4} t}
\end{aligned}
$$


for all $t>0$ and for $K$ large enough so that $2 \rho_{0} \leq a$. Hence

$$
\mathbb{E}_{n}\left[e^{\rho_{0} T_{n_{* *}(K)}} \mathbb{1}_{\left\{T_{n_{* *}(K)}<\frac{t}{2}\right\}}\right]=\frac{\varphi_{n}}{\varphi_{n_{* *}(K)}}+\mathcal{O}(1) K e^{-\frac{a}{4} t}
$$

where we used the identity $\mathbb{E}_{n}\left[e^{\rho_{0} T_{n_{* *}(K)}}\right]=\frac{\varphi_{n}}{\varphi_{n_{* *}(K)}}$ for all $n \geq n_{* *}(K)$. This identity comes from the fact that the process

$$
\left(e^{\rho_{0}\left(t \wedge T_{n_{* *}(K)}\right)} \varphi\left(X_{t \wedge T_{n_{* *}(K)}^{K}}^{K}\right), t \geq 0\right)
$$

is a martingale (where we write $\varphi(n)$ instead of $\varphi_{n}$ for the sake of readability). This relies on the equation $L \varphi=-\rho_{0} \varphi$. The identity then follows from the Martingale Stopping Theorem (see e.g. 23]). Therefore we obtain

$$
\sup _{A \in \mathscr{P}\left(\mathbb{N}^{*}\right)}\left|P_{t}(n, A)-e^{-\rho_{0} t} \varphi_{n} \frac{\langle\varphi, \mathbb{1}\rangle_{\pi}}{\|\varphi\|_{\pi}^{2}} \nu(A)\right| \leq \mathcal{O}(1)\left(K e^{-\frac{a}{4} t}+K^{\frac{3}{4}} e^{c_{1} K} e^{-\frac{\rho_{1}}{2} t}\right)
$$

for all $n \geq n_{* *}(K)$. The same bound holds for all $n<n_{* *}(K)$ using Proposition 7.2 .

\subsection{Proof of Theorem 3.6}

We first establish inequality (3.7). Observe that for every $B \in \mathscr{P}(\mathbb{N})$

$$
\begin{aligned}
\mathbb{P}_{n}\left(X_{t}^{K} \in B\right) & =\mathbb{P}_{n}\left(X_{t}^{K} \in B \cap \mathbb{N}^{*}\right)+\mathbb{P}_{n}\left(X_{t}^{K} \in B \cap\{0\}\right) \\
& =\mathbb{P}_{n}\left(X_{t}^{K} \in B \cap \mathbb{N}^{*}\right)+\delta_{0}(B)\left(1-\mathbb{P}_{n}\left(X_{t}^{K} \in \mathbb{N}^{*}\right)\right) .
\end{aligned}
$$

Inequality (3.7) follows by using twice Proposition 7.3. This implies the first inequality in the theorem using Proposition 7.1. Theorem 3.2 and statement 3 in Proposition 5.1

The second inequality in the theorem is proved as follows. Let $t_{1}(K)$ be such that for all $t \geq t_{1}(K)$

$$
\sup _{n \geq 1} \frac{\|\varphi\|_{\pi}^{2}}{\varphi_{n}\langle\varphi, \mathbb{1}\rangle_{\pi}} C^{\prime}\left(K e^{-\frac{a}{4} t}+K^{\frac{3}{4}} e^{c_{1} K} e^{-\frac{\rho_{1}}{2} t}\right) \leq \frac{1}{2} .
$$

We start by considering $t \geq t_{1}(K)$. We have using Proposition 7.3

$$
\begin{aligned}
\left|\frac{P_{t}(n, A)}{P_{t}\left(n, \mathbb{N}^{*}\right)}-\nu(A)\right| & =\left|\frac{P_{t}(n, A)-\nu(A) P_{t}\left(n, \mathbb{N}^{*}\right)}{P_{t}\left(n, \mathbb{N}^{*}\right)}\right| \\
& \leq \frac{2\|\varphi\|_{\pi}^{2} e^{\rho_{0} t}}{\varphi_{n}\langle\varphi, \mathbb{1}\rangle_{\pi}}\left|P_{t}(n, A)-\nu(A) P_{t}\left(n, \mathbb{N}^{*}\right)\right| .
\end{aligned}
$$

The bound follows using again Proposition 7.3 Proposition 7.1, Lemma 9.3 (twice), Theorem 3.2 and Propositions 5.1 and 5.2. To have the bound for all $t<t_{1}(K)$, observe that the left-hand side is at most equal to 2 . The bound follows by eventually taking a larger constant (uniformly in $n, K$ and $t$ ). 


\section{Proof of Theorem 3.7}

Let $K$ be large enough such that $n_{1}=n_{*}(K)-\sqrt{K} \log K>0$ and $n_{2}=$ $n_{*}(K)+\sqrt{K} \log K<n_{* *}(K)$. We have

$$
\frac{\pi_{n}}{\pi_{n_{*}(K)}}= \begin{cases}\frac{\mu_{n_{*}(K)}}{\mu_{n}} \Lambda_{n, n_{*}(K)}^{-1}, & \text { for } \quad n \geq n_{*}(K), \\ \frac{\mu_{n}}{\mu_{n_{*}(K)}} \Lambda_{n_{*}(K), n}, & \text { for } \quad n \leq n_{*}(K) .\end{cases}
$$

For $n \leq n_{1}, \Lambda_{n_{*}(K), n}$ is increasing, $\mu_{n} \leq \mathcal{O}(1) K$ and $\mu_{n_{*}(K)} \geq 1$ ( $K$ large). Therefore using Lemma 9.4 we get

$$
\sum_{n=1}^{n_{1}} \frac{\pi_{n}}{\pi_{n_{*}(K)}} \leq \mathcal{O}(1) K^{2} e^{-c(\log K)^{2}}
$$

Using Lemma 9.3. Propositions 5.2 and [5.1, and Theorem 3.2 this implies

$$
\sum_{n=1}^{n_{1}} \frac{\pi_{n} \varphi_{n}}{\pi_{n_{*}(K)} \varphi_{n_{*}(K)}} \leq \mathcal{O}(1) K^{2} e^{-c(\log K)^{2}} .
$$

For $n_{2} \leq n \leq n_{* *}(K), \Lambda_{n, n_{*}(K)}^{-1}$ is decreasing, $\mu_{n} \leq \mathcal{O}(1) K$ and $\mu_{n_{*}(K)} \geq 1(K$ large), therefore using Lemma 9.4 we have (since $H^{\prime \prime}\left(x_{*}\right)>0$ )

$$
\sum_{n=n_{2}}^{n_{* *}(K)} \frac{\pi_{n}}{\pi_{n_{*}(K)}} \leq \mathcal{O}(1) K^{2} e^{-c(\log K)^{2}} .
$$

For $n \geq n_{* *}(K)$ we have

$$
\Lambda_{n, n_{*}(K)}^{-1} \leq \Lambda_{n_{* *(K), n *(K)}}^{-1}\left(\frac{1}{2}\right)^{n-n_{* *(K)}}
$$

hence

$$
\sum_{n=n_{* *}(K)}^{\infty} \frac{\pi_{n}}{\pi_{n_{*}(K)}} \leq \mathcal{O}(1) K^{2} e^{-c(\log K)^{2}} .
$$

Using Lemma 9.3 , Propositions 5.2 and 5.1 and Theorem 3.2 this implies

$$
\sum_{n=n_{2}}^{\infty} \frac{\pi_{n} \varphi_{n}}{\pi_{n_{*}(K)} \varphi_{n_{*}(K)}} \leq \mathcal{O}(1) K^{2} e^{-c(\log K)^{2}}
$$

Finally, for $n_{*}(K) \leq n \leq n_{2}$, using Lemma 9.4 we have

$$
\begin{aligned}
\frac{\pi_{n}}{\pi_{n_{*}(K)}} & =\frac{\mu_{n_{*}(K)}}{\mu_{n}} \sqrt{\frac{\lambda_{n_{*}(K)}}{\mu_{n_{*}(K)}} \frac{\mu_{n}}{\lambda_{n}}} e^{-K\left(H\left(\frac{n}{K}\right)-H\left(\frac{n_{*}(K)}{K}\right)\right)-\frac{c\left(n_{*}(K), n, K\right)}{K}} \\
& =e^{-\frac{\left(n-n_{*}(K)\right)^{2}}{2 K \sigma^{2}}}\left(1+\frac{\mathcal{O}(1)}{K}\left(n-n_{*}(K)\right)+\frac{\mathcal{O}(1)}{K^{2}}\left(n-n_{*}(K)\right)^{3}\right) .
\end{aligned}
$$


The same estimate holds for $n_{1} \leq n \leq n_{*}(K)$.

It is easy to verify using Lemma 9.3 , Propositions 5.2 and 5.1, Theorem 3.2 and Lemma 9.4 that for $n_{1} \leq n \leq n_{2}$

$$
\sup _{n_{1} \leq n \leq n_{2}}\left|1-\frac{\varphi_{n}}{\varphi_{n_{*}(K)}}\right| \leq \frac{\mathcal{O}(1)}{K^{2}}
$$

This implies for $n_{1} \leq n \leq n_{2}$

$$
\begin{aligned}
& \frac{\pi_{n} \varphi_{n}}{\pi_{n_{*}(K)} \varphi_{n_{*}(K)}} \\
& =e^{-\frac{\left(n-n_{*}(K)\right)^{2}}{2 K \sigma^{2}}}\left(1+\frac{\mathcal{O}(1)}{K^{2}}\left(n-n_{*}(K)\right)^{3}+\frac{\mathcal{O}(1)}{K}\left(n-n_{*}(K)\right)+\frac{\mathcal{O}(1)}{K^{2}}\right) .
\end{aligned}
$$

Therefore, setting

$$
g_{K}(n)=e^{-\frac{\left(n-n_{*}(K)\right)^{2}}{2 K \sigma^{2}}}
$$

we obtain

$$
\sum_{n=n_{1}}^{n_{2}}\left|\frac{\pi_{n} \varphi_{n}}{\pi_{n_{*}(K)} \varphi_{n_{*}(K)}}-g_{K}(n)\right| \leq \mathcal{O}(1) .
$$

We also observe that

$$
\sum_{n=1}^{n_{1}} g_{K}(n)+\sum_{n=n_{2}}^{\infty} g_{K}(n) \leq \mathcal{O}(1) \sqrt{K} e^{-\tilde{c}(\log K)^{2}}
$$

for some positive constant $\tilde{c}$. Theorem 3.7 follows after some easy manipulations of the normalizations.

\section{Appendix: some technical lemmas and estimates}

Let $I=\int_{\frac{x_{*}}{2}}^{+\infty} \frac{\mathrm{d} x}{x \tilde{\mu}(x)}$. Recall that we assume that $I<+\infty$ (see (2.6) $)$.

LEMMA 9.1.

There exists $C \geq 1$ such that for all $K>1$

$$
\sum_{p=n_{*}(K)+1}^{\infty} \frac{1}{\mu_{p}} \leq I \quad \text { and } \quad \sum_{j=n_{*}(K)}^{\infty} \frac{1}{\lambda_{j} \pi_{j}} \sum_{p=j+1}^{\infty} \pi_{p} \leq C K .
$$

Proof. Using (2.1) we get

$$
\sum_{p=n_{*}(K)+1}^{\infty} \frac{1}{\mu_{p}}=\frac{1}{K} \sum_{p=n_{*}(K)+1}^{\infty} \frac{1}{\frac{p}{K} \tilde{\mu}\left(\frac{p}{K}\right)} \leq \int_{\frac{n_{*}(K)}{K}}^{\infty} \frac{\mathrm{d} x}{x \tilde{\mu}(x)} \leq I .
$$


This proves the first estimate. Next, by definition of $n_{*}(K), n_{* *}(K)$ and $x_{* *}$ (see Section 2 and Subsection 6.2), we have

$$
\begin{aligned}
\sum_{j=n_{*}(K)}^{\infty} \sum_{p=j+1}^{\infty} \frac{1}{\lambda_{j}} \frac{\pi_{p}}{\pi_{j}} & =\sum_{j=n_{*}(K)}^{n_{* *}(K)} \sum_{p=j+1}^{\infty} \frac{1}{\lambda_{j}} \frac{\pi_{p}}{\pi_{j}}+\sum_{j=n_{* *}(K)+1}^{\infty} \sum_{p=j+1}^{\infty} \frac{1}{\lambda_{j}} \frac{\pi_{p}}{\pi_{j}} \\
& \leq \sum_{j=n_{*}(K)}^{n_{* *}(K)} \sum_{p=j+1}^{\infty} \frac{1}{\mu_{p}}+\sum_{j=n_{* *}(K)+1}^{\infty} \sum_{p=j+1}^{\infty}\left(\frac{1}{2}\right)^{p-j} \frac{1}{\mu_{p}} \\
& \leq n_{* *}(K) I+\sum_{p=n_{* *}(K)+1}^{\infty} \frac{1}{\mu_{p}} \leq n_{* *}(K) I+\sum_{p=n_{*}(K)+1}^{\infty} \frac{1}{\mu_{p}} \\
& \leq\left(x_{* *} K+1\right) I \leq C K
\end{aligned}
$$

where we set $C=\left(x_{* *}+1\right) I$ and where we used Young's inequality to get the second inequality.

LEMMA 9.2.

The quantity

$$
a=\inf _{K>1}\left(\sum_{j=n_{* *}(K)}^{\infty} \frac{1}{\lambda_{j} \pi_{j}} \sum_{p=j+1}^{\infty} \pi_{p}\right)^{-1}
$$

where $n_{* *}(K)$ is defined in (6.7), is strictly positive.

Proof. The proof follows immediately from the above proof noticing that

$$
\sum_{j=n_{* *}(K)+1}^{\infty} \sum_{p=j+1}^{\infty} \frac{1}{\lambda_{j}} \frac{\pi_{p}}{\pi_{j}} \leq \sum_{p=n_{*}(K)+1}^{\infty} \frac{1}{\mu_{p}} \leq I .
$$

Recall that $u^{0}$ is defined in (3.3).

\section{LEMMA 9.3.}

There exists a constant $C>0$ such that for all $K$ large enough, and for all $1 \leq n \leq n_{*}(K)$

$$
1 \leq u_{n}^{0} \leq C .
$$

Proof. We take $K$ large enough such that

$$
1<\left\lfloor K x_{* * *}\right\rfloor<\left\lfloor K x_{*}\right\rfloor-2
$$


where $x_{* * *}$ is defined in (6.8). Observe that $u_{n}^{0}$ is increasing hence for $n \leq$ $n_{*}(K)$

$$
\begin{aligned}
u_{n}^{0} & \leq u_{n_{*}(K)}^{0} \\
& \leq 1+\sum_{j=1}^{n_{* * *}(K)-1} \frac{1}{\lambda_{j} \pi_{j}}+\sum_{j=n_{* * *}(K)}^{n_{*}(K)} \frac{1}{\lambda_{j} \pi_{j}} \\
& \leq 1+\frac{1}{1-\theta}+\left(n_{*}(K)-n_{* * *}(K)+1\right) \theta^{n_{* * *}(K)} \leq C
\end{aligned}
$$

where $C>0$ is independent of $K$.

For $n>m$ let

$$
\Lambda_{n, m}=\prod_{j=m}^{n-1} \frac{\mu_{j}}{\lambda_{j}}
$$

By convention we set $\Lambda_{n, n}=1$. We have the following lemma.

\section{LEMMA 9.4.}

For all $m, n \in \mathbb{N}^{*}$ such that $n>m$ we have

$$
\Lambda_{n, m}=\sqrt{\frac{\mu_{m}}{\lambda_{m}} \frac{\lambda_{n}}{\mu_{n}}} e^{K\left(H\left(\frac{n}{K}\right)-H\left(\frac{m}{K}\right)\right)+\frac{c(m, n, K)}{K}}
$$

where $H$ is defined in (2.8) and where $\sup _{m, n, K}|c(m, n, K)|<\infty$.

Proof. By definition (2.1)

$$
\Lambda_{n, m}=\prod_{j=m}^{n-1} \frac{\tilde{\mu}\left(\frac{j}{K}\right)}{\tilde{\lambda}\left(\frac{j}{K}\right)}=e^{\sum_{j=m}^{n-1} h\left(\frac{j}{K}\right)}
$$

where $h(s):=\log (\tilde{\mu}(s) / \tilde{\lambda}(s))\left(H^{\prime}(s)=h(s)\right)$. Using the trapezoidal rule we get

$$
\begin{aligned}
& \log \Lambda_{n, m} \\
& =\frac{1}{2}\left(h\left(\frac{n}{K}\right)-h\left(\frac{m}{K}\right)\right)+K\left(H\left(\frac{n}{K}\right)-H\left(\frac{m}{K}\right)\right)+\frac{1}{12 K^{2}} \sum_{j=m}^{n-1} h^{\prime \prime}\left(\frac{\xi_{j}}{K}\right)
\end{aligned}
$$

for some $\xi_{j} \in[j, j+1]$. Therefore, using (2.9), we obtain

$\log \Lambda_{n, m}=\frac{1}{2}\left(h\left(\frac{n}{K}\right)-h\left(\frac{m}{K}\right)\right)+K\left(H\left(\frac{n}{K}\right)-H\left(\frac{m}{K}\right)\right)+\frac{1}{K} c(m, n, K)$.

The results follows. 


\section{LEMMA 9.5.}

For all $K>1$ we have $u_{n_{*}(K)}^{0}=\frac{1}{1-\frac{\mu_{1}}{\lambda_{1}}}+\mathcal{O}\left(\frac{1}{K}\right)$.

Proof. We have

$$
\begin{aligned}
u_{n_{*}(K)}^{0} & =1+\sum_{j=1}^{n_{*}(K)-1} \frac{1}{\lambda_{j} \pi_{j}} \\
& =1+\sum_{j=1}^{\lfloor\sqrt{K}\rfloor} \prod_{\ell=1}^{j} \frac{\tilde{\mu}\left(\frac{\ell}{K}\right)}{\tilde{\lambda}\left(\frac{\ell}{K}\right)}+\sum_{j=\lfloor\sqrt{K}\rfloor+1}^{n_{*}(K)-1} \prod_{\ell=1}^{j} \frac{\tilde{\mu}\left(\frac{\ell}{K}\right)}{\tilde{\lambda}\left(\frac{\ell}{K}\right)} .
\end{aligned}
$$

The first sum (plus 1) is equal to

$$
\begin{aligned}
& 1+\sum_{j=1}^{\sqrt{K}}\left(\frac{\tilde{\mu}(0)}{\tilde{\lambda}(0)}\right)^{j} e^{\mathcal{O}(1) \frac{j^{2}}{K}} \\
& =1+\sum_{j=1}^{\sqrt{K}}\left(\frac{\tilde{\mu}(0)}{\tilde{\lambda}(0)}\right)^{j}+\mathcal{O}\left(\frac{1}{K} \sum_{j=1}^{\sqrt{K}} j^{2}\left(\frac{\tilde{\mu}(0)}{\tilde{\lambda}(0)}\right)^{j}\right) \\
& =\frac{1}{1-\frac{\mu_{1}}{\lambda_{1}}}+\mathcal{O}\left(\frac{1}{K}\right) .
\end{aligned}
$$

The second sum is bounded similarly and we get

$$
\mathcal{O}(1) K\left(\frac{\tilde{\mu}(0)}{\tilde{\lambda}(0)}\right)^{\sqrt{K}} \leq \frac{\mathcal{O}(1)}{K} .
$$

The lemma is proved.

The next lemma is about estimating various quantities: $u_{n_{*}(K)}^{0}-u_{n_{*}(K)-1}^{0}$ (where $u_{n}^{0}$ is defined in (3.3)), $W_{n_{*}(K)}^{0}$ (see (5.5) for the definition), $\Delta_{n_{*}(K)}^{0}$ $\Delta_{n_{*}(K)-1}^{0}\left(\right.$ where $\Delta_{n}^{0}$ is defined in (5.2) ) and $D(K)$ (that is defined in (5.9) ).

\section{LEMMA 9.6.}

For all $K>1$ we have the following estimates.
1. $u_{n_{*}(K)}^{0}-u_{n_{*}(K)-1}^{0}=\sqrt{\frac{\mu_{1}}{\lambda_{1}}} e^{K \int_{\frac{1}{K}}^{\frac{n_{*}(K)}{K}} \log \frac{\tilde{\mu}(x)}{\lambda(x)} \mathrm{d} x}\left(1+\frac{\mathcal{O}(1)}{K}\right) ;$
2. $W_{n_{*}(K)}^{0}=\frac{\sqrt{2 \pi}}{2 x_{*} \tilde{\lambda}\left(x_{*}\right) \sqrt{K H^{\prime \prime}\left(x_{*}\right)}}\left(1+\frac{(\log K)^{3}}{\sqrt{K}}\right)$;
3. $\Delta_{n_{*}(K)}^{0}-\Delta_{n_{*}(K)-1}^{0}=-\frac{\sqrt{2 \pi}}{2 x_{*} \tilde{\lambda}\left(x_{*}\right) \sqrt{K H^{\prime \prime}\left(x_{*}\right)}}\left(1+\frac{(\log K)^{3}}{\sqrt{K}}\right)$; 


$$
\text { 4. } D(K)=\frac{1}{1-\frac{\mu_{1}}{\lambda_{1}}} \frac{\sqrt{2 \pi}}{x_{*} \tilde{\lambda}\left(x_{*}\right) \sqrt{K H^{\prime \prime}\left(x_{*}\right)}}\left(1+\frac{(\log K)^{3}}{\sqrt{K}}\right) \text {; }
$$

5. There exist a constant $\gamma \in(0,1)$, that is independent of $K$, such that

$$
\gamma \sqrt{K} e^{c K} \leq \sum_{j=1}^{\infty} \pi_{j} \leq \gamma^{-1} \sqrt{K} e^{c K},
$$

where $c$ is defined in (3.4).

Proof. The proof of the first statement follows from Lemma 9.4, namely

$$
\begin{aligned}
u_{n_{*}(K)}^{0}-u_{n_{*}(K)-1}^{0} & =\frac{1}{\lambda_{n_{*}(K)-1} \pi_{n_{*}(K)-1}}=\Lambda_{n_{*}(K), 1} \\
& =\sqrt{\frac{\mu_{1} \lambda_{n_{*}(K)}}{\lambda_{1} \mu_{n_{*}(K)}}} e^{K\left(H\left(\frac{n_{*}(K)}{K}\right)-H\left(\frac{1}{K}\right)\right)+\frac{c\left(1, n_{*}(K), K\right)}{K}} \\
& =\sqrt{\frac{\mu_{1}}{\lambda_{1}}} e^{K \int_{\frac{n_{*}(K)}{K}}^{\frac{1}{K}} \log \frac{\tilde{\mu}(x)}{\bar{\lambda}(x)} \mathrm{d} x}\left(1+\frac{\mathcal{O}(1)}{K}\right) .
\end{aligned}
$$

We continue by estimating $W_{n_{*}(K)}^{0}$. Write

$$
W_{n_{*}(K)}^{0}=\sum_{p=n_{*}(K)}^{\infty} \frac{\pi_{p}}{\lambda_{n_{*}(K)-1} \pi_{n_{*}(K)-1}}=I_{1}+I_{2}+I_{3} .
$$

We start by estimating $I_{3}$. We again make use of Lemma 9.4 .

$$
\begin{aligned}
I_{3} & =\sum_{p=n_{* *}(K)}^{\infty} \frac{\pi_{p}}{\lambda_{n_{*}(K)-1} \pi_{n_{*}(K)-1}}=\sum_{p=n_{* *(K)}}^{\infty} \frac{1}{\mu_{p}} \prod_{j=n_{*}(K)}^{n_{* *(K)}-1} \frac{\lambda_{j}}{\mu_{j}} \prod_{j=n_{* *}(K)}^{p-1} \frac{\lambda_{j}}{\mu_{j}} \\
& \leq \frac{\mathcal{O}(1)}{K} \sum_{p=n_{* *}(K)}^{\infty}\left(\frac{1}{2}\right)^{\left.n-n_{* *}(K)\right)} \leq \frac{\mathcal{O}(1)}{K}
\end{aligned}
$$

using the monotonicity of $\left(\mu_{n}\right)_{n}$ and the definition of $n_{* *}(K)$.

We now estimate $I_{2}$.

$$
\begin{aligned}
& I_{2}=\sum_{p=n_{*}(K)+\sqrt{K} \log K+1}^{n_{* *(K)}-1} \frac{\pi_{p}}{\lambda_{n_{*}(K)-1} \pi_{n_{*}(K)-1}}=\sum_{p=n_{*}(K)+\sqrt{K} \log K+1}^{n_{* *}(K)-1} \frac{1}{\mu_{p} \Lambda_{p, n_{*}(K)}} \\
& =\sum_{p=n_{*}(K)+\sqrt{K} \log K+1}^{n_{* *}(K)-1} \frac{1}{\mu_{p}} \sqrt{\frac{\mu_{p} \lambda_{n_{*}(K)}}{\lambda_{p} \mu_{n_{*}(K)}}} e^{-K\left(H\left(\frac{p}{K}\right)-H\left(\frac{n_{*}(K)}{K}\right)\right)}\left(1+\frac{\mathcal{O}(1)}{K}\right) \\
& \leq \sum_{p=n_{*}(K)+\sqrt{K} \log K+1}^{n_{* *}(K)-1} \frac{1}{\sqrt{\lambda_{p} \mu_{p}}} e^{-K\left(H\left(\frac{n_{*}(K)}{K}+\frac{\log K}{\sqrt{K}}\right)-H\left(\frac{n_{*}(K)}{K}\right)\right)}\left(1+\frac{\mathcal{O}(1)}{K}\right) \\
& \leq \frac{\mathcal{O}(1)}{K}\left(n_{* *}(K)-n_{*}(K)\right) e^{-\frac{(\log K)^{2} H^{\prime \prime}\left(x_{*}\right)}{2}+\frac{\mathcal{O}(1)(\log K)^{3}}{\sqrt{K}}} \\
& \leq \mathcal{O}(1) e^{-\frac{(\log K)^{2} H^{\prime \prime}\left(x_{*}\right)}{2}}
\end{aligned}
$$


using the monotonicity of $H$ and Taylor's expansion.

Finally we estimate $I_{1}$. We use again Lemma 9.4 .

$$
\begin{aligned}
I_{1} & =\sum_{p=n_{*}(K)}^{n_{*}(K)+\sqrt{K} \log K} \frac{\pi_{p}}{\lambda_{n_{*}(K)-1} \pi_{n_{*}(K)-1}} \\
& =\sum_{p=n_{*}(K)}^{n_{*}(K)+\sqrt{K} \log K} \frac{1}{\mu_{p}} \sqrt{\frac{\mu_{p} \lambda_{n_{*}(K)}}{\lambda_{p} \mu_{n_{*}(K)}}} e^{-K\left(H\left(\frac{p}{K}\right)-H\left(\frac{n_{*}(K)}{K}\right)\right)\left(1+\frac{\mathcal{O}(1)}{K}\right)} \\
& =\sum_{p=n_{*}(K)}^{n_{*}(K)+\sqrt{K} \log K} \frac{1}{\sqrt{\lambda_{p} \mu_{p}}} e^{-\frac{H^{\prime \prime}\left(x_{*}\right)\left(p-n_{*}(K)\right)^{2}}{2 K}}\left(1+\frac{\mathcal{O}(1)(\log K)^{3}}{\sqrt{K}}\right) \\
& =\int_{x_{*}}^{x_{*}+\frac{\log K}{\sqrt{K}}} \frac{1}{x \sqrt{\tilde{\lambda}(x) \tilde{\mu}(x)}} e^{-\frac{K H^{\prime \prime}\left(x_{*}\right)\left(x-x_{*}\right)^{2}}{2}} \mathrm{~d} x\left(1+\frac{(\log K)^{3}}{\sqrt{K}}\right) \\
& =\frac{1}{2} \frac{\sqrt{2 \pi}}{\sqrt{K H^{\prime \prime}\left(x_{*}\right)}} \frac{1}{x_{*} \tilde{\lambda}\left(x_{*}\right)}\left(1+\frac{(\log K)^{3}}{\sqrt{K}) .}\right.
\end{aligned}
$$

The estimation of

$$
\Delta_{n_{*}(K)}^{0}-\Delta_{n_{*}(K)-1}^{0}=-\sum_{p=1}^{n_{*}(K)-1} \frac{\left(u_{p}^{0}\right)^{2} \pi_{p}}{\lambda_{n_{*}(K)-1} \pi_{n_{*}(K)-1} u_{n_{*}(K)-1}^{0} u_{n_{*}(K)}^{0}} .
$$

is done similarly by decomposing the sum into three sums with the same ranges as before.

The estimation for $D(K)$ follows immediately from the above estimates and Lemma 9.5

Finally, the upper bound in statement 5 is obtained as follows. We have

$$
\sum_{n=1}^{\infty} \pi_{n}=\sum_{n=1}^{\infty} \frac{1}{\mu_{n} \Lambda_{n, 1}}
$$

where $\Lambda_{n, 1}=\prod_{j=1}^{n-1} \frac{\mu_{j}}{\lambda_{j}}$. Using Lemma 9.4 we get

$$
\begin{aligned}
\sum_{n=1}^{\infty} \pi_{n} & =\sum_{n=1}^{n_{* *(K)}} \pi_{n}+\sum_{n=n_{* *}(K)+1}^{\infty} \pi_{n} \\
& \leq \mathcal{O}(1) \sum_{n=1}^{n_{* *(K)}} \frac{1}{\sqrt{\lambda_{n} \mu_{n}}} e^{-K\left(H\left(\frac{n}{K}\right)-H\left(\frac{1}{K}\right)\right)}+\sum_{n=n_{* *}(K)+1}^{\infty} \pi_{n} .
\end{aligned}
$$

The second sum is estimated by using the fact that $\lambda_{j} / \mu_{j}<1 / 2$ for $j \geq$ $n_{* *}(K)$. The first sum is split into a sum from 1 to $n_{* * *}(K)$ and a sum from $n_{* * *}(K)+1$ to $n_{* *}(K)$. In both cases, we use Lemma 9.4 and the steepest 
descent method for the sum from $n_{* * *}(K)+1$ to $n_{* *}(K)$. The lower bound in statement 5 is obtained using

$$
\sum_{n=1}^{\infty} \pi_{n} \geq \sum_{n=n_{* * *}(K)}^{n_{* * *}(K)} \pi_{n}
$$

and the steepest descent method as before. This finishes the proof of the lemma.

Consider the linear equations

$$
\alpha_{n} w_{n+1}+\beta_{n} w_{n-1}-\left(\alpha_{n}+\beta_{n}\right) w_{n}=h_{n}
$$

where $\left(\alpha_{n}\right)_{n \geq 1},\left(\beta_{n}\right)_{n \geq 1}$ and $\left(h_{n}\right)_{n \geq 1}$ are given sequences of real numbers. The coefficients $\alpha_{n}$ and $\beta_{n}$ are positive. Define

$$
\Theta_{p, q}=\prod_{j=q}^{p-1} \frac{\beta_{j}}{\alpha_{j}} \quad \text { for } p>q \text { and } \quad \Theta_{q, q}=1 .
$$

Note that for $r \geq s \geq q$

$$
\Theta_{r, s}=\frac{\Theta_{r, q}}{\Theta_{s, q}}
$$

We have the following lemma.

\section{LEMMA 9.7.}

The general solution of the homogeneous equation (9.1) when $h_{n}=0$ for all $n \geq 1$ (homogeneous equation) satisfies the recurrence property

$$
w_{n}=w_{q}+\left(w_{q+1}-w_{q}\right) \sum_{j=q}^{n-1} \Theta_{j+1, q+1}, \forall n \geq q .
$$

In the general case, the solution of (9.1) is

$$
w_{n}=w_{q}+\left(w_{q+1}-w_{q}\right) \sum_{j=q}^{n-1} \Theta_{j+1, q+1}+\sum_{j=q}^{n-1} \sum_{p=q+1}^{j} \frac{h_{p}}{\alpha_{p}} \Theta_{j+1, p+1} .
$$

In case of convergence of $\sum_{p=q}^{\infty} \frac{h_{p}}{\alpha_{p} \Theta_{p+1, q}}$, this can be rewritten as

$$
w_{n}=w_{q}+\tilde{A}_{q} \sum_{j=q}^{n-1} \Theta_{j+1, q}-\sum_{j=q}^{n-1} \sum_{p=j+1}^{\infty} \frac{h_{p}}{\alpha_{p} \Theta_{p+1, j+1}}, \forall n \geq q
$$

for some constant $\tilde{A}_{q}$. (We use the convention $\sum_{q}^{q-1}=0$.) 
Proof. For $n \geq q$ we define $A_{n+1}$ by

$$
w_{n+1}-w_{n}=A_{n+1} \Theta_{n+1, q}
$$

Then

$$
\alpha_{n} A_{n+1} \Theta_{n+1, q}-\beta_{n} A_{n} \Theta_{n, q}=\alpha_{n} \Theta_{n+1, q}\left(A_{n+1}-A_{n}\right)=h_{n}
$$

i.e.

$$
A_{n+1}-A_{n}=\frac{h_{n}}{\alpha_{n} \Theta_{n+1, q}}
$$

and for all $n \geq q+1$

$$
A_{n}=A_{q}+\sum_{j=q}^{n-1} \frac{h_{j}}{\alpha_{j} \Theta_{j+1, q}} \quad \text { with } \sum_{q}^{q-1}=0
$$

where

$$
A_{q}=\frac{w_{q+1}-w_{q}}{\Theta_{q+1, q}}-\frac{h_{q}}{\alpha_{q} \Theta_{q+1, q}} .
$$

Then for all $n \geq q$

$w_{n+1}-w_{n}=A_{q} \Theta_{n+1, q}+\Theta_{n+1, q} \sum_{j=q}^{n} \frac{h_{j}}{\alpha_{j} \Theta_{j+1, q}}=A_{q} \Theta_{n+1, q}+\sum_{j=q}^{n} \frac{h_{j}}{\alpha_{j}} \Theta_{n+1, j+1}$.

Hence

$$
w_{n}=w_{q}+A_{q} \sum_{j=q}^{n-1} \Theta_{j+1, q}+\sum_{j=q}^{n-1} \sum_{p=q}^{j} \frac{h_{p}}{\alpha_{p}} \Theta_{j+1, p+1} .
$$

This implies the first two statements of the lemma. In case of convergence this can be rewritten as

$$
w_{n}=w_{q}+\tilde{A}_{q} \sum_{j=q}^{n-1} \Theta_{j+1, q}-\sum_{j=q}^{n-1} \sum_{p=j+1}^{\infty} \frac{h_{p}}{\alpha_{p} \Theta_{p+1, j+1}}
$$

for some constant $\tilde{A}_{q}$. Indeed, since $j \geq p \geq q$, we have $\Theta_{j+1, p+1}=\frac{\Theta_{j+1, q}}{\Theta_{p+1, q}}$. Thus

$$
\begin{aligned}
\sum_{j=q}^{n-1} \sum_{p=q}^{j} \frac{h_{p}}{\alpha_{p}} \Theta_{j+1, p+1} & =\sum_{j=q}^{n-1} \Theta_{j+1, q} \sum_{p=q}^{j} \frac{h_{p}}{\alpha_{p} \Theta_{p+1, q}} \\
& =\sum_{j=q}^{n-1} \Theta_{j+1, q}\left(\sum_{p=q}^{\infty} \frac{h_{p}}{\alpha_{p} \Theta_{p+1, q}}-\sum_{p=j+1}^{\infty} \frac{h_{p}}{\alpha_{p} \Theta_{p+1, q}}\right)
\end{aligned}
$$

which implies the last statement of the lemma. 
Acknowledgments. The third author benefited from the support of the "Chaire Modélisation Mathématique et Biodiversité" funded by Veolia Environnement, the Ecole polytechnique and the Muséum national d'Histoire naturelle. The authors thank the referees for their careful reading and comments.

\section{References}

[1] L. J. S. Allen. An introduction to stochastic processes with applications to biology. CRC Press, 2011.

[2] A. D. Barbour, P. K. Pollett. Total variation approximation for quasistationary distributions. J. Appl. Probab. 47 (2010), 934-946.

[3] V. Bansaye, S. Méléard, M. Richard. How do birth and death processes come down from infinity? Preprint, 2013 (arXiv:1310.7402 [math.PR]).

[4] P. Cattiaux, P. Collet, A. Lambert, S. Martínez, S. Méléard, J. San Martín. Quasi-stationary distributions and diffusion models in population dynamics. Ann. Probab. 37 (5) (2009), 1926-1969.

[5] N. Champagnat, D. Villemonais. Exponential convergence to quasi-stationary distribution and Q-process. Preprint, 2014 (arXiv:1404.1349v1 [math.PR]).

[6] B. Cloez, M.N. Thai. Quantitative results for the Fleming-Viot particle system in discrete space. Preprint, 2014 (arXiv:1312.2444v2 [math.PR]).

[7] P. Collet, S. Martínez, J. San Martín. Quasi-Stationary Distributions. Probability and its Applications. Springer, 2013.

[8] P. Diaconis, L. Miclo. On quantitative convergence to quasi-stationarity. Preprint, 2014 (arXiv:1406.1805v1 [math.PR]).

[9] C. Doering, K. Sargsyan, L. Sander. Extinction times for birth-death processes: exact results, continuum asymptotics, and the failure of the Fokker-Planck approximation. Multiscale Model. Simul. 3 (2005), no. 2, 283-299.

[10] E. van Doorn. Quasi-stationary distributions and convergence to quasistationarity for birth-death processes. Adv. Appl. Probab. 23 (1991), 683-700.

[11] M. Fedoryuk. Asymptotic analysis. Linear ordinary differential equations. Springer-Verlag, Berlin, 1993. 
[12] S. Karlin, J. L. McGregor. The differential equations of birth and death processes and the Stieltjes moment problem. Trans. Amer. Math. Soc. 86 (1957), 489-546.

[13] S. Karlin, H. M. Taylor. An introduction to stochastic modeling. Third edition. Academic Press, 1998.

[14] T. Kato. Perturbation Theory of Linear Operators. Springer, 1966.

[15] D. Kessler, N. Shnerb. Extinction rates for fluctuation-induced metastabilities: a real-space WKB approach. J. Stat. Phys. 127 (2007), no. 5, $861-886$.

[16] T. G. Kurtz. Solutions of ordinary differential equations as limits of pure jump Markov processes. J. Appl. Probab. 7, 49-58.

[17] N. Levinson. The asymptotic nature of solutions of linear systems of differential equations. Duke Math. J. 15 (1948), 111-126.

[18] S. Méléard, D. Villemonais. Quasi-stationary distributions and population processes. Probab. Surv. 9 (2012), 340-410.

[19] I. Nåsell. Extinction and quasi-stationarity in the stochastic logistic SIS model. Lecture Notes in Mathematics 2022. Mathematical Biosciences Subseries. Springer (2011).

[20] O. Ovaskainen, B. Meerson. Stochastic models of population extinction. Trends Ecol Evol. 25 (2010), 643-652.

[21] S. Sagitov, A. Shahmerdenova. Extinction times for a birth-death process with weak competition. Lithuanian Math. Journal. 53 (2013), 220234.

[22] J. Sotomayor. Inversion of smooth mappings. Z. Angew. Math. Phys. 41 (1990), no. 2, 306-310.

[23] D. Revuz, M. Yor. Continuous martingales and Brownian motion. Fundamental Principles of Mathematical Sciences 293. Springer-Verlag, Berlin, 1991.

[24] K. Yosida. Functional analysis. Reprint of the sixth (1980) edition. Classics in Mathematics. Springer-Verlag, Berlin, 1995. 